\title{
Dielectric Properties of Phase Separated Blends containing a Microcapacitor Network of Carbon Nanotubes: Compatibilization by a Random or Block Copolymer
}

\author{
Avanish Bharati ${ }^{a}$, Michael Wübbenhorst ${ }^{b}$, Paula Moldenaers ${ }^{\mathrm{a}}$ and Ruth \\ Cardinaels $^{\mathrm{a}, \mathrm{c}}$ \\ aSoft Matter Rheology and Technology, Department of Chemical Engineering, KU Leuven, \\ Celestijnenlaan 200F, P.B. 2424, B-3001 Leuven, Belgium \\ bSoft Matter and Biophysics Section, Department of Physics and Astronomy, KU Leuven, \\ Celestijnenlaan 200D, P.B. 2416, B-3001 Leuven, Belgium \\ 'Polymer Technology, Department of Mechanical Engineering, TU Eindhoven, P.B. 513, \\ 5600 MB Eindhoven, The Netherlands \\ E-mail: paula.moldenaers@cit.kuleuven.be \\ Phone: +32 16 32-23-59
}

Final manuscript after referee comments

Manuscript published in Macromolecules

Please cite as: Bharati A, Wübbenhorst M, Moldenaers P, Cardinaels R Dielectric Properties of Phase Separated Blends containing a Microcapacitor Network of Carbon Nanotubes: Compatibilization by a Random or Block Copolymer, 2017, 50 (10), pp 3855-3867 Macromolecules (2017)

The published manuscript can be found at:

http://pubs.acs.org/doi/abs/10.1021/acs.macromol.6b02786 


\section{Dielectric Properties of Phase Separated Blends} containing a Microcapacitor Network of Carbon Nanotubes: Compatibilization by a Random or Block Copolymer

Avanish Bharati, ${ }^{\dagger}$ Michael Wübbenhorst, ${ }^{\ddagger}$ Paula Moldenaers, ${ }^{*} \dagger$ and Ruth

$$
\text { Cardinaels } \mathbf{s}^{\dagger,}
$$

$\dagger$ Soft Matter Rheology and Technology, Department of Chemical Engineering, KU Leuven, Celestijnenlaan 200F, P.B. 2424, B-3001 Leuven, Belgium

$\ddagger$ Soft Matter and Biophysics Section, Department of Physics and Astronomy, KU Leuven, Celestijnenlaan 200D, P.B. 2416, B-3001 Leuven, Belgium

\Polymer Technology, Department of Mechanical Engineering, TU Eindhoven, P.B. 513, 5600 MB Eindhoven, The Netherlands

E-mail: paula.moldenaers@cit.kuleuven.be

Phone: +32 16 32-23-59 


\section{Abstract}

The mechanisms governing the dielectric blend properties at different length scales for 2 phase separating blends with multiwall carbon nanotubes (MWNTs) are unravelled by 3 tuning the microstructure. Thereto, compatibilization by interfacially segregated block 4 copolymers (bcp) and random copolymers (rcp) of poly(styrene-random/block-methyl 5 methacrylate)(PS-r/b-PMMA) was achieved in phase separating blends of poly[( $\alpha$-methyl 6 styrene)-co-acrylonitrile] and poly(methyl methacrylate) (P $\alpha$ MSAN/PMMA) undergoing 7 spinodal decomposition. In our recent work, we elucidated the effects of copolymer ar- 8 chitecture and molecular weight on the percolating network of selectively localized MWNTs. 9 Only short bcp and long rep/bcp improved the connectivity and refinement of the P $\alpha \mathrm{MSAN} \quad 10$ phase laden with MWNTs and the resulting conductivity. In the present work, we study the ${ }_{11}$ effects of copolymer type, architecture and concentration on the dielectric properties. We ${ }_{12}$ demonstrate a concurrent increase of the interfacial capacitance and decrease of the interfa- ${ }_{13}$ cial resistance of MWNTs with entrapped P $\alpha$ MSAN upon effective compatibilization. This ${ }_{14}$ is attributed to the increasing amount of connected parallel microcapacitor RC elements 15 formed by the network of adjacent MWNTs enclosing a thin dielectric layer of P $\alpha$ MSAN. $\quad 16$ At high frequencies (above $1 \mathrm{MHz}$ ) the electrons hop between the neighboring MWNTs, ${ }_{17}$ whereas at intermediate frequencies, the electrons of the MWNTs tunnel through the barri- ${ }_{18}$ ers imposed by the entrapped P $\alpha \mathrm{MSAN}$. The physical characteristics of the microcapacitor 19 network namely the thickness of the microcapacitors and the volume fraction of entrapped 20 P $\alpha$ MSAN contributing to the microcapacitor network are estimated by describing the di- ${ }_{21}$ electric relaxation time and strength using the fluctuation induced tunneling model and the 22 interlayer model respectively. Combining the knowledge of the aforementioned parameters ${ }_{23}$ allows to describe the evolution of the total interfacial capacitance of the microcapacitor ${ }_{24}$ assembly as a function of copolymer type and concentration. Our robust and simple pro- ${ }_{25}^{25}$ cedure to tune the MWNT microcapacitor network in polymer blends via the efficiency of ${ }_{26}$ the compatibilizer can be used to achieve a synergistic increase in the dielectric properties ${ }_{27}$ 
at different length scales.

\section{Keywords:}

Polymer blend, phase separation, carbon nanotubes, compatibilization, interfacial capaci- 3 tance, microcapacitor, dielectric properties.

\section{Introduction}

When developing immiscible polymer blends with enhanced properties, block ${ }^{1}$ and random copolymers $^{2}$ play an essential role in refining and stabilizing the biphasic morphology. 7 The copolymers architecture ${ }^{3}$ and molecular weight ${ }^{4}$ govern its interfacial activity and 8 its resulting efficiency in compatibilizing the blend morphology. The copolymers physical 9 characteristics affect morphology refinement differently under flow and quiescent conditions 10 encountered respectively during melt mixing and thermal annealing of immiscible blends. ${ }^{5} \quad{ }_{11}$ In phase separating blends, the extent of symmetry of the copolymers strongly affects the 12 miscibility range, ${ }^{6}$ which further alters the quench depth and the phase separation kinetics. ${ }_{13}$ In addition, both the copolymer type and its molecular weight govern its ability to reach 14 the interface and to stabilize the biphasic morphology against coarsening. ${ }^{7}$ Copolymers 15 with various physical characteristics can thus be used to create blend systems with a wide 16 range of morphologies at the same blend composition.

Varying the compatibilizers efficiency can be used to tailor the blend morphology and to 18 improve the resulting blend properties. The consequences of an effective compatibilization 19 for the physical properties of biphasic blends such as mechanical or dielectric behavior 20 have been studied. For example, the mechanical properties of immiscible blends can be ${ }_{21}^{21}$ significantly enhanced upon compatibilization due to improved adhesion of the blend 22 components $^{8}{ }^{8}$ with the efficiency being correlated with the compatibilizers composition ${ }^{9}$ and ${ }_{23}$ architecture. ${ }^{3}$ Long random copolymers can be more effective than long symmetric diblock $\quad 24$ 
copolymers in reinforcing interfaces. ${ }^{2}$ This has been attributed to the ability of the long 1 random copolymers to have multiple crossings at the interface, forming loops which entangle 2 with the homopolymers and, thereby, effectively stitch the two immiscible homopolymers 3 together. ${ }^{2}$ Recently, it was shown that an increase in the molar mass of the PMMA end 4 blocks in PMMA-PE-PMMA triblock copolymers results in an increase of the mechanical 5 properties of PE-PMMA blends, which was attributed to an enhanced entanglement 6 density and increased interfacial width at the PE/PMMA interface. ${ }^{10}$ Similarly, maximum 7 fracture toughness of the interface between the components of an immiscible PS-PMMA 8 blend resulted from a symmetrical interfacial broadening due to PS-r-PMMA. ${ }^{11}$ From the 9 perspective of blends dielectric properties, differences in the dielectric and/or conducting 10 properties of the blend components further lead to interfacial blocking of charge carriers. ${ }^{12}{ }_{11}$ An understanding of the consequences of effective compatibilization for the interfacial 12 dynamics and the associated length scales would allow to develop polymeric materials 13 with enhanced dielectric properties. Recently, we have studied the effect of an interfacially 14 segregated PS-r-PMMA random copolymer on the interfacial dynamics of a phase separated 15 blend of P $\alpha$ MSAN and PMMA. ${ }^{13}$ The interfacially segregated compatibilizer simultaneously 16 affected the blocking of charge carriers at the interface and refined the dispersed phase in $\quad 17$ which the charge entrapment occurred. The complex interplay of the respective length 18 scales determined the final blend dielectric properties. ${ }^{13} \quad 19$ In blends with selectively localized conductive nanoparticles, compatibilizers can also 20 enhance the blend conductivity as a consequence of their effect on the blend morphology. ${ }^{7,14}{ }_{21}^{21}$ For example, in phase separating P $\alpha$ MSAN/PMMA blends with MWNTs, an interfacially 22 segregated long random copolymer was used to steer the formation of a percolated network ${ }_{23}$ of MWNTs in the P $\alpha$ MSAN phase, which resulted in an increase of the blend conductivity ${ }_{24}$ by 4 decades with $0.5 \mathrm{wt} \%$ MWNTs upon addition of only $0.25 \mathrm{wt} \%$ copolymer. ${ }^{15} \mathrm{We}{ }^{25}$ recently demonstrated that varying the copolymers architecture (block or random) and 26 molecular weight can be used to tune the phase separated blend morphology and corre- ${ }_{27}$ 
sponding conductivity. ${ }^{7}$ In polymer nanocomposites with conductive particles, not only the 1 conductivity, but also the dielectric properties have been studied to some extent. Polymer 2 nanocomposites with high dielectric permittivity provide an ideal solution to combine the 3 dielectric and conducting properties of the ceramic or metal fillers and the mechanical 4 properties of the polymer matrix. As a result, these dielectric materials find tremendous 5 application in electromechanical devices, supports of electronic circuits, cable accessories in 6 electrical engineering and in many civilian and military applications. ${ }^{16}$ Specifically dielectric 7 polymer materials are embedded into the inner layers of organic printed circuit boards 8 (PCB) because of their flexibility and good compatibility with the PCB material, for which 9 it is essential for the composites to exhibit a low percolation threshold. ${ }^{17}$ In this context, ${ }_{10}$ the present work aims to improve the dielectric properties of MWNT filled polymer blends ${ }_{11}$ at a low MWNT concentration.

Nearby conductive particles enclosing a layer of dielectric polymer form a microcapacitor, 13 with the particles forming the capacitor electrodes. These microcapacitor configurations ${ }_{14}$ are not perfect capacitors but can be modeled as a resistor and capacitor in parallel. ${ }^{18}$ Such $\quad{ }_{15}$ microcapacitors can provide extremely high dielectric permittivities to the materials. ${ }^{19}$ For $\quad{ }_{16}$ rubber composites with MWNTs, the relaxation process of the MWNT/MWNT connections $\quad{ }_{17}$ exhibits the characteristics of a tunneling process. Using this framework, the resistivity ${ }_{18}$ and gap distance of the microcapacitors was calculated from the dielectric properties. A 19 pronounced decrease in both the resistivity and gap distance of the microcapacitor was 20 obtained with increasing MWNT concentration. ${ }^{18}$ Furthermore, the interfacial polarization ${ }_{21}$ of MWNTs in a polyvinyl alcohol composite was modeled using an equivalent circuit 22 consisting of resistor-capacitor elements in series allowing to decouple the bulk behavior ${ }_{23}$ from that of the electrode-sample interface. ${ }^{20}$ At present, the effect of a microcapacitor ${ }^{24}$ network in one of the blend phases on the blend dielectric properties and the effect of 25 the morphology on the latter is unknown. The goal of our research is to address the ${ }_{26}$ consequence of compatibilization of the blend on the microcapacitor network formed by ${ }_{27}$ 
entrapped P $\alpha$ MSAN between adjacent MWNTs at the same MWNT concentration. The 1 interfacial properties along with the mechanism of charge transfer of the MWNTs through 2 the entrapped P $\alpha$ MSAN will be studied. This will allow to elucidate the effect of various 3 copolymers on the physical parameters governing the dielectric properties of the blends 4 and their length scales. A systematic understanding of the filler-filler, filler-matrix polymer 5 and filler-entrapped polymer-filler interactions and their associated length scales will allow 6 to tailor physical properties of the blends by an effective compatibilization. Our findings will be pivotal for developing a rational design of cost-effective high performance biphasic 8 polymer blends with improved dielectric properties.

\section{Experimental Section}

\subsection{Materials}

The homopolymers poly[( $\alpha$-methyl styrene)-co-acrylonitrile] (P $\alpha$ MSAN) (Luran KR2556) ${ }_{13}$ and poly(methyl methacrylate) (PMMA) (Lucryl G77) were purchased from BASF. The ${ }_{14}$ P $\alpha$ MSAN-PMMA blend is miscible at low temperatures and phase separates by spinodal 15 decomposition upon heating above the lower critical solution temperature (LCST) at 16 $165{ }^{\circ} \mathrm{C}$ for blends containing $40 \mathrm{wt} \% \mathrm{P} \alpha \mathrm{MSAN} .{ }^{21}$ Amine functionalized multiwall carbon ${ }_{17}$ nanotubes (MWNTs) were purchased from Nanocyl (NC3152). It is well-known that these 18 MWNTs selectively localize in the P $\alpha$ MSAN phase, which is their thermodynamically 19 preferred phase. ${ }^{7,15,22}$ The phase separated morphology is compatibilized by either random 20 or block poly(styrene)-poly(methyl methacrylate) (PS-PMMA) copolymers with various 21 molecular weights. The physical characteristics of the blend components and copolymers 22 chosen in the study are compiled in Table 1 . The set of copolymers has been chosen to cover ${ }_{23}$ a wide range of compatibilization efficiencies, corresponding to different levels of interfacial ${ }_{24}$ activity, as shown by Bharati et al. ${ }^{7}$ 
Table 1. Physical characteristics of the blend components and copolymers.

\begin{tabular}{lllll}
\hline Component & Company & $\mathrm{M}_{w}(\mathrm{~kg} / \mathrm{mol})$ & $\mathrm{M}_{w} / \mathrm{M}_{n}$ & Composition $\left(\mathrm{M}_{w}(\mathrm{~kg} / \mathrm{mol})\right)$ \\
\hline P $\alpha$ MSAN & BASF (Luran KR2556) & 82.0 & 2.4 & $70 \%$ P $\alpha$ MS 30\% PAN \\
PMMA & BASF & 88.0 & 2.1 & $95 \%$ PMMA, 5\% PMA \\
PS-r-PMMA & Polymer Source & 33.8 & 1.3 & $\mathrm{M}_{w}^{P S}=13.5, \frac{M_{w}^{P M M A}}{M_{w}^{P S}}=1.5$ \\
PS-r-PMMA & Polymer Source & 192.0 & 1.6 & $\mathrm{M}_{w}^{P S}=61.5, \frac{M_{w}^{P M M A}}{M_{w}^{P S}}=2.1$ \\
PS-r-PMMA & Polysciences & 270.0 & 5.0 & $\mathrm{M}_{w}^{P S}=189.0, \frac{M_{w}^{P S} M^{P S}}{M^{P S}}=0.4$ \\
PS-b-PMMA & Polymer Source & 36.8 & 1.18 & $\mathrm{M}_{w}^{P S}=18.4, \frac{M_{w}^{P M} \Psi^{P S}}{M_{w}^{P S}}=1.0$ \\
PS-b-PMMA & Polymer Source & 291.0 & 1.18 & $\mathrm{M}_{w}^{P S}=145.5, \frac{M_{w}^{P M M A}}{M_{w}^{P S}}=1.0$ \\
\hline
\end{tabular}

\subsection{Blend processing}

One-step melt mixing was used to prepare 40/60 (wt\%/wt\%) P $\alpha$ MSAN/PMMA blends 3 with or without 0.5 wt\% MWNTs and 0, 0.25, 0.5 and 2 wt\% PS-PMMA copolymers. A 4 $15 \mathrm{~cm}^{3}$ DSM microcompounder with a rotational speed of $60 \mathrm{rpm}$ for $20 \mathrm{~min}$ was used 5 under inert nitrogen conditions at $200{ }^{\circ} \mathrm{C}$. P $\alpha$ MSAN nanocomposites with a concentration 6 of MWNTs (1.25 wt\%) equivalent to that in the blends taking into account the selective 7 localization in the P $\alpha \mathrm{MSAN}$, were extruded under similar conditions. Due to the torque 8 limitations of the mini-extruder, the blend processing was performed at a temperature 9 $\left(200{ }^{\circ} \mathrm{C}\right)$ above the LCST of the $40 / 60 \mathrm{P} \alpha \mathrm{MSAN} / \mathrm{PMMA}$ blend $\left(165{ }^{\circ} \mathrm{C}\right)$, resulting in 10 phase separated blends. Subsequently, these blends were compression molded below the ${ }_{11}$ $\operatorname{LCST}\left(\right.$ at $160{ }^{\circ} \mathrm{C}$ ) for $8 \mathrm{~min}$ under $50 \mathrm{MPa}$ using a laboratory press (Collin). This 12 resulted in monophasic blends, as verified by Bose et al. ${ }^{22}$ using DMTA. Phase separation ${ }_{13}$ of the compression molded samples (25 $\mathrm{mm}$ diameter and $1.5 \mathrm{~mm}$ thickness) was per- ${ }_{14}$ formed under nitrogen atmosphere at $220{ }^{\circ} \mathrm{C}$ in a rheo-dielectric setup for 5 hrs. After ${ }_{15}$ this time, both the elastic moduli as well as the conductivity had reached steady state values. $\quad 16$ 


\subsection{Characterizations}

The dielectric measurements were performed with a rheo-dielectric setup by employing a 2 Novocontrol Alpha analyzer in combination with an MCR501 stress controlled rheometer 3 (Anton Paar). Measurements were performed on the phase separated samples $(25 \mathrm{~mm} \quad 4$ diameter and $1.5 \mathrm{~mm}$ thickness), placed in between the parallel plates of the rheometer, 5 which acted as electrodes for dielectric measurements. Dielectric spectroscopy measurements 6 were conducted in the frequency range of $10^{-2}$ to $10^{7} \mathrm{~Hz}$. High temperatures were main- 7 tained with a CTD450 temperature controlled convection oven under nitrogen atmosphere. 8 Temperatures below as well as above the phase separation temperature were used. For 9 temperatures below the phase separation temperature, measurements were performed above $\quad 10$ the components glass transition temperature $\left(\mathrm{T}_{g}\right)$, which allowed good electrical contact. $\quad 11$ The dielectric scans at each temperature were performed on different samples and the 12 absence of remixing was verified by DSC. ${ }^{13}$ The data were analyzed by calculating the ${ }_{13}$ dielectric permittivity and the real part of the conductivity from the complex impedance 14 values and the sample dimensions. ${ }^{12,23}$ The contribution of conductivity to the dielectric ${ }_{15}$ loss $\left(\epsilon^{\prime \prime}\right)$ was eliminated by calculating $\epsilon^{\prime \prime}$ from the dielectric constant $\left(\epsilon^{\prime}\right)$ using $\mathrm{d} \epsilon^{\prime} / \mathrm{d} \ln f$ as $\quad{ }_{16}$ an approximation of the Kramers-Kronig (KK) transformation. ${ }^{23}$ The Nyquist impedance ${ }_{17}$ spectra were fitted with an appropriate equivalent circuit using ZVIEW2 software to 18 simulate the experimental impedance response. 19 


\section{Results and discussion}

\subsection{MWNT-P $\alpha$ MSAN Interfacial Capacitance}

\subsubsection{Characterization of the Dynamics and Origin of the Interfacial Peak}

In our recent work, we elucidated the effects of copolymer architecture and molecular weight $\quad 4$ in improving the connectivity and refinement of the P $\alpha$ MSAN phase with MWNTs in phase separated P $\alpha$ MSAN-PMMA blends. ${ }^{15}$ In the present work, we will probe the interfacial dy- 6 namics of the blends with MWNTs and copolymer to evaluate the effects of an improved microstructure on the interfacial dielectric properties. Interfacial dielectric relaxations arise due to a contrast in electrical permittivity and/or conductivity of the different constituents. ${ }^{12,24}$ The interfacial dynamics in blends with MWNTs and rcp may involve the cumulative effect of the polarization of various interfaces, namely that of the more conducting P $\alpha$ MSAN with the less conducting rcp and that of the selectively localized conducting MWNTs with enclosed dielectric P $\alpha$ MSAN chains. Here, we will first attempt to identify the presence and origin of such an interfacial peak in compatibilized blends with selectively localized MWNTs. Thereto, the interfacial relaxations in the compatibilized biphasic P $\alpha$ MSAN-PMMA blends with MWNTs will be compared with the dynamics of the P $\alpha$ MSAN at the rcp interface in the neat biphasic blend (without MWNTs) as well as that of the conducting MWNTs with the entrapped dielectric P $\alpha \mathrm{MSAN}$ chains in a P $\alpha \mathrm{MSAN}$ composite having an equivalent concentration of MWNTs (1.25 wt\% MWNTs) as in the blends (0.5 wt\% MWNTs in 40 wt $\%$ P $\alpha$ MSAN).

The "conduction free" dielectric relaxation spectra of the homopolymers and biphasic blends 21 with $0.5 \mathrm{wt} \% \mathrm{rcp}$ of $270 \mathrm{~kg} / \mathrm{mol}$ with and without $0.5 \mathrm{wt} \%$ MWNTs near the constituents ${ }_{22}$ $\mathrm{T}_{g}\left(130{ }^{\circ} \mathrm{C}\right)$ are shown in Figure $1 \mathrm{a}$ and b, respectively. The conductivity contributions ${ }_{23}$ originating from the blend components above their $\mathrm{T}_{g}$ as well as from the MWNT network ${ }^{24}$ were removed for blends without and with MWNTs. The dielectric spectra of the blend ${ }_{25}$ components as well as P $\alpha$ MSAN/PMMA blends with and without copolymer have been de- $\quad 26$ 
scribed in detail elsewhere ${ }^{13}$ and will only be discussed briefly here. In the "conduction free" dielectric relaxation spectra of the homopolymers in Figure 1a, a high frequency $(10 \mathrm{kHz} 2$ - $1 \mathrm{MHz}$ ) $\beta$ relaxation is observed in the homopolymers, which also appears in the blends 3 and composites (Figure 1b). The stronger $\beta$ relaxation in PMMA is attributed to the co- ${ }_{4}$ ordinated rotation of the $-\mathrm{COOCH}_{3}$ side groups around the backbone chain of PMMA. ${ }^{25,26}{ }_{5}$ At intermediate frequencies $(1 \mathrm{~Hz}-10 \mathrm{kHz})$, a segmental $(\alpha)$ relaxation is discerned in the 6 homopolymers, and its contribution in the blends (Figure 1b) is owed to both the blend 7 components. Considering the higher dielectric strength and slower dynamics of the $\alpha$ relax- 8 ation in P $\alpha$ MSAN as compared to that of PMMA due to the stronger cooperative motions 9 of the backbone chain of $\mathrm{P} \alpha \mathrm{MSAN}$, we believe the $\alpha$ relaxations of $\mathrm{P} \alpha \mathrm{MSAN}$ are primarily $\quad 10$ manifested in the blends and composites. At low frequency (below $1 \mathrm{~Hz}$ ) an increase in ${ }_{11}$ the "conduction free" dielectric relaxation spectra is observed in biphasic blends with and 12 without MWNTs, which has a higher dielectric strength and slower relaxation as compared 13 to the segmental and molecular relaxations in Figure 1a. Such a strong low frequency relax- ${ }_{14}$ ation peak is typically of interfacial origin. ${ }^{13}$

At low frequencies, the "conduction free" dielectric relaxation spectra of the homopolymers in Figure 1a also increase with decreasing frequency (with a slope less than 1) due to weak 17 electrode polarization effects. For the blends and composites the low frequency relaxation 18 peak is more pronounced. Moreover, it was unaffected by performing measurements at a 19 reduced sample thickness of $\approx 400 \mu \mathrm{m}$ (as compared to $1.5 \mathrm{~mm}$ ), which is uncharacteristic $\quad 20$ of electrode polarization. ${ }^{23}$ Hence, the low frequency relaxation in the blend without the ${ }_{21}$ MWNTs is of an interfacial origin and results from the entrapment of occluded P $\alpha$ MSAN 22 charges $\left(\sigma_{d c} \approx 10^{-13} \mathrm{~S} / \mathrm{cm}\right.$ at $\left.130{ }^{\circ} \mathrm{C}\right)$ at the interface with less conducting interfacially seg- ${ }_{23}$ regated copolymer $\left(\sigma_{d c} \approx 10^{-15} \mathrm{~S} / \mathrm{cm}\right.$ at $\left.130^{\circ} \mathrm{C}\right)$. Moreover, with increasing concentration 24 of the copolymer, an increase of the dielectric strength of the interfacial polarization was 25 observed, resulting from a decrease of the Debye length. ${ }^{13}$ In summary, a low frequency and 26 high temperature dielectric relaxation peak of interfacial origin is observed in the biphasic ${ }_{27}$ 

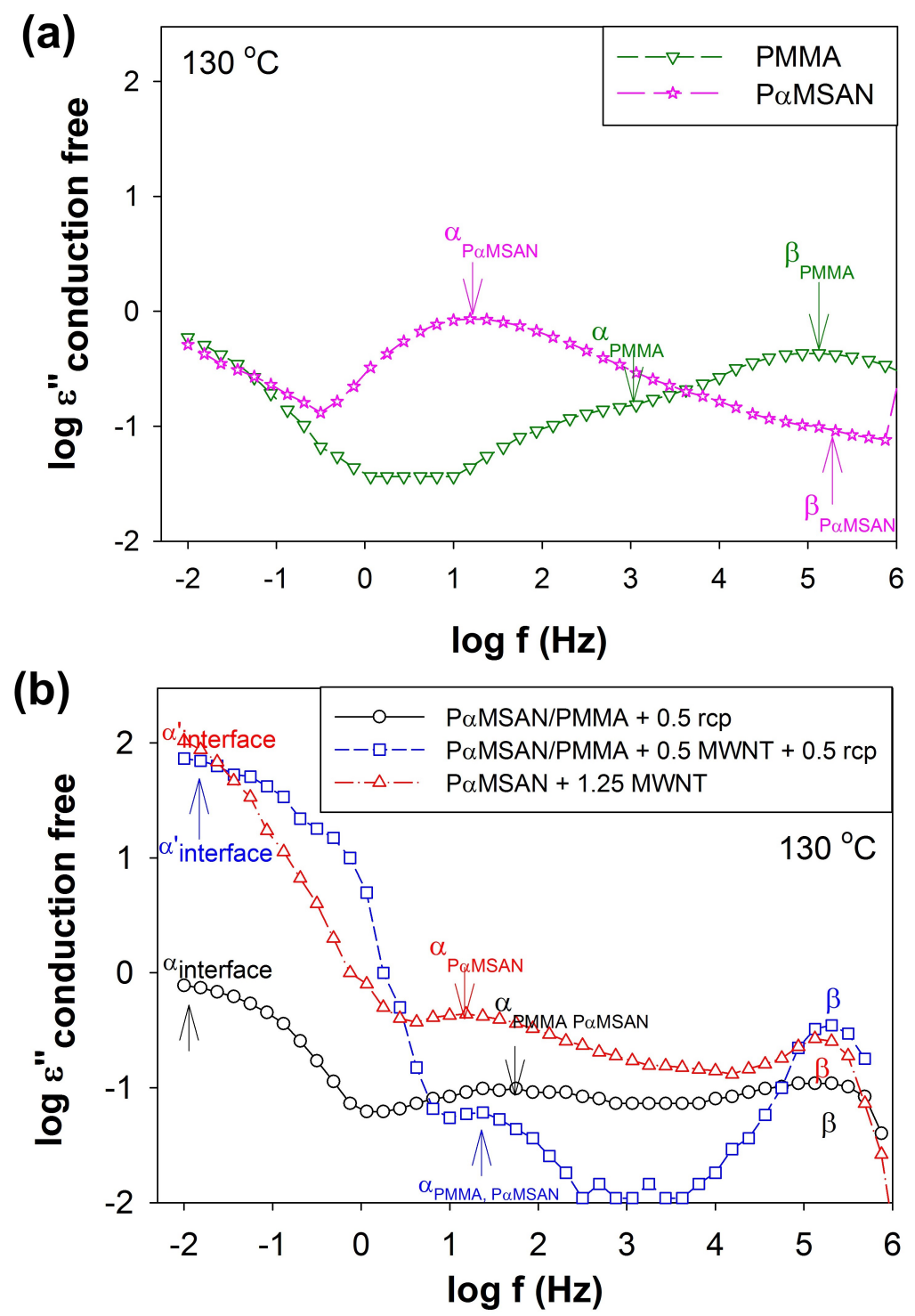

Figure 1. Comparison of "conduction free" dielectric loss spectra of (a) homopolymers PMMA and P $\alpha$ MSAN, (b) biphasic 40/60/0.5 P $\alpha$ MSAN/PMMA/rcp $270 \mathrm{~kg} / \mathrm{mol}$ blends with and without $0.5 \mathrm{wt} \%$ MWNTs, and P $\alpha$ MSAN composite with $1.25 \mathrm{wt} \%$ MWNTs above the $\mathrm{T}_{g}$ of the homopolymers $\left(130{ }^{\circ} \mathrm{C}\right)$. The indicated peaks are assigned to their respective contributions. $\alpha_{\text {interface }}$ originates from the polymer-polymer interface, whereas $\alpha_{\text {interface }}^{\prime}$ originates from the MWNT-polymer interface.

blend without MWNTs due to a conductivity contrast of 2 decades between the dispersed 1 polar P $\alpha$ MSAN and the interfacially segregated copolymer.

Upon addition of $0.5 \mathrm{wt} \%$ MWNTs, the biphasic blend with $0.5 \mathrm{wt} \% \mathrm{rcp}$ also reveals a 3 
low frequency polarization peak in Figure 1b. However, its dielectric strength is two decades larger than the strength of the interfacial polarization in the biphasic blend without MWNTs. 2 Since it is established that the dielectric strength in these blends increases more or less pro- 3 portional with the intrinsic length scale of the biphasic blend, i.e. the ratio of structure 4 dimension $\left(\mathrm{D}_{v}\right)$ to Debye length $\left(\mathrm{L}_{D}\right),{ }^{13}$ we cannot attribute the two decades increase of the 5 dielectric strength to the marginal refinement effects achieved from the $0.5 \mathrm{wt} \%$ MWNTs. ${ }^{7} \quad 6$ As the MWNTs are preferentially segregated only in the P $\alpha$ MSAN phase, the possibility of 7 probing the interface between the MWNTs and entrapped P $\alpha$ MSAN cannot be ruled out. $\quad 8$ Therefore, the loss spectrum of a P $\alpha \mathrm{MSAN}$ nanocomposite with an equivalent concentration 9 of MWNTs as in the blend (1.25 wt\% MWNTs) has been included in Figure 1b to study 10 the dynamics of the interface of entrapped P $\alpha$ MSAN chains between neighboring MWNTs. ${ }_{11}$ Interestingly, a stark resemblance of the dielectric strength of the interfacial polarization of 12 MWNTs-P $\alpha$ MSAN in the composite and in the blend offers substantial support to the fact 13 that we are probing the same interface in the biphasic blend with MWNTs. As the P $\alpha$ MSAN ${ }_{14}$ composite with $1.25 \mathrm{wt} \%$ MWNTs is inherently not percolating, ${ }^{27}$ the presence of electrode ${ }^{15}$ polarization in the composite at low frequencies can be ruled out, which was also verified 16 by the unchanged dielectric strength of the low frequency relaxation at a sample thickness ${ }_{17}$ of $400 \mu \mathrm{m}$ as compared to $1.5 \mathrm{~mm}$. In conclusion, a substantial increase of the dielectric re- ${ }_{18}$ laxation strength by over 2 decades in magnitude at low frequencies is observed for biphasic 19 blends with $0.5 \mathrm{wt} \% \mathrm{rcp}$ upon addition of $0.5 \mathrm{wt} \%$ MWNTs, whose origin is attributed to 20 the polarization of the MWNTs charges at the interface with entrapped P $\alpha$ MSAN chains. ${ }_{21}^{21}$ To further verify the origin of the dielectric interfacial polarization at low frequencies, its 22 dynamics in the studied composites and blends is investigated as a function of temperature ${ }_{23}$ in Figure 2. Thereto, the dielectric interfacial relaxation peak was fitted using the Havriliak 24 Negami (HN) function: ${ }^{28}$

$$
\begin{array}{r}
\epsilon^{\prime \prime}(\omega)=\operatorname{Im}\left(\sum \frac{\Delta \epsilon}{\left[1+\left(\iota \omega \tau_{H N}\right)^{a}\right]^{b}}\right) \\
\Delta \epsilon=\epsilon_{s}-\epsilon_{\infty}
\end{array}
$$


where $\epsilon_{\infty}$ is the dielectric constant at limiting high frequencies, $\tau_{H N}$ is the characteristic relaxation time, and $a$ and $b$ are the shape parameters, characteristic of the width and 2 peak asymmetry of the relaxation time distribution function, respectively. The dielectric 3 strength and relaxation time of the interfacial polarization obtained using the HN function 4 for temperatures above the $\mathrm{T}_{g}$ of the homopolymers $\left(130{ }^{\circ} \mathrm{C}\right)$ and below the phase separation $\quad 5$ temperature of the blend $\left(160{ }^{\circ} \mathrm{C}\right)$ are plotted in Figure 2. The interfacial relaxation time 6 shows a slight deviation from a linear temperature dependence, and hence has been fitted 7 using the Vogel-Fulcher-Tammann (VFT) function ${ }^{29-31}$ (lines in Figure 2):

$$
\tau_{\max }=\tau_{\infty} \exp \frac{E_{v}}{R\left(T-T_{v}\right)}
$$

where $\tau_{\infty}$ is the pre-exponential factor and corresponds to the relaxation time at the limit of 9 infinite temperature, $R$ is the universal gas constant, $E_{v}$ is the "Vogel activation energy" in 10 $\mathrm{kJ} / \mathrm{mol}$ and $T_{v}$ is the Vogel temperature, below which the system is frozen and relaxation is 11 absent. This non-linear temperature dependence of the interfacial relaxation time points to a 12 spatial gradient of the properties of entrapped P $\alpha$ MSAN chains between adjacent MWNTs. $\quad 13$ Upon comparison of the temperature dependence of the interfacial relaxation times in the 14 blends and composites in Figure 2, it can be concluded that the difference in the temperature 15 dependence of the interfacial relaxation time in the three systems is insignificant. Impera- 16 tively, a close agreement between the relaxation strength of the biphasic blend with MWNTs 17 and that of the P $\alpha$ MSAN nanocomposite with an equivalent concentration of MWNTs is 18 observed for the entire temperature window. In summary, a striking similarity of the dielec- 19 tric interfacial strength and relaxation time of the MWNTs-P $\alpha$ MSAN interface both in the 20 blends and the composites over the entire temperature window corroborates our finding that $\quad 21$ we are studying the dynamics of the MWNTs-P $\alpha$ MSAN interface in the biphasic compat- 22 ibilized blend at low frequencies. In the ensuing sections, we will estimate the capacitance ${ }_{23}$ of the dielectric interfacial relaxations of the MWNTs-P $\alpha$ MSAN network and will further ${ }_{24}$ 


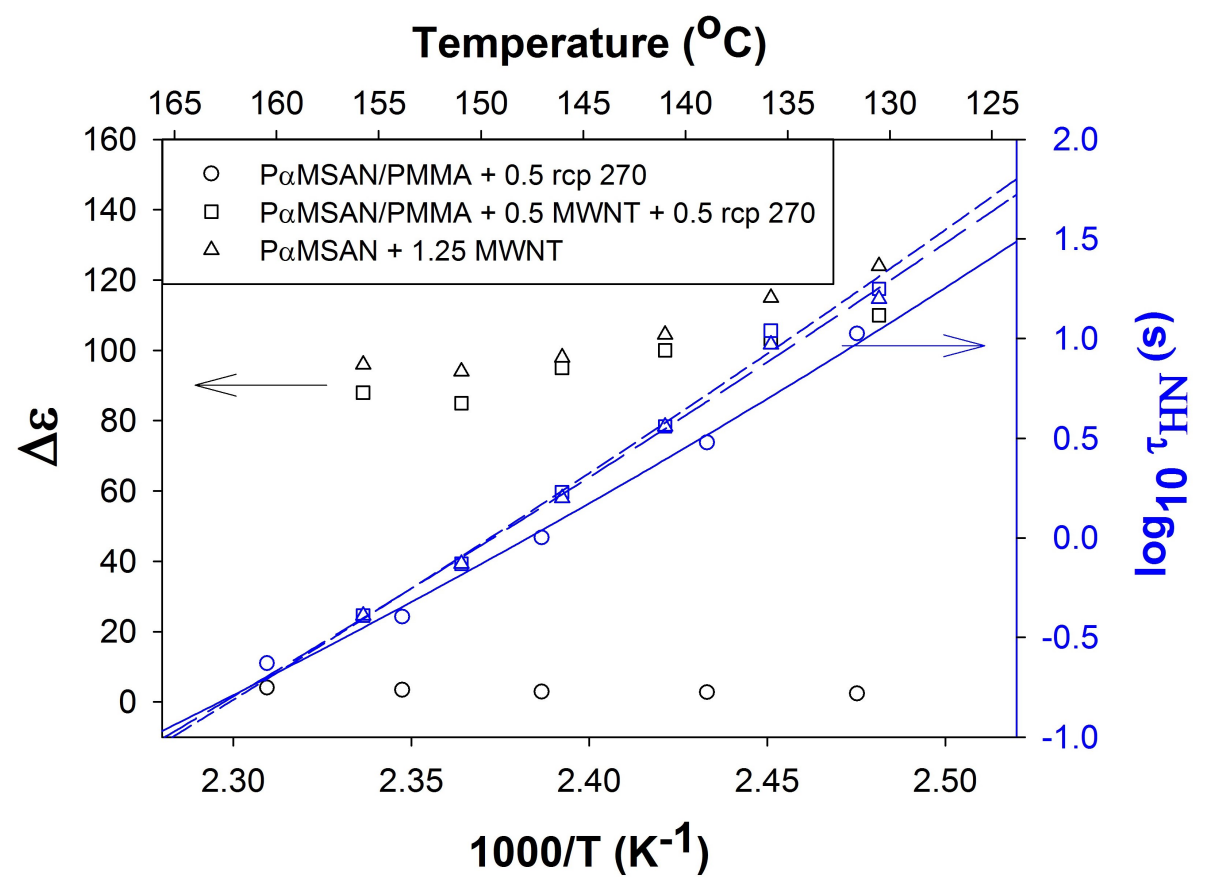

Figure 2. Temperature dependence of the dielectric strength $\Delta \epsilon$ (left) and relaxation time (right) determined from the Havriliak Negami $(\mathrm{HN})$ function for interfacial polarization of biphasic 40/60/0.5 P $\alpha$ MSAN/PMMA/rcp $270 \mathrm{~kg} / \mathrm{mol}$ blends with and without $0.5 \mathrm{wt} \%$ MWNTs and PoMSAN composite with 1.25 wt\% MWNTs.

investigate the effect of various copolymers on the estimated capacitance.

\subsubsection{Determination of Interfacial Capacitance by means of Impedance Spec-} troscopy

The dynamic response of the biphasic blend may allow to quantify the effect of copolymer on properties at different length scales. In this regard, we demonstrated the ability of an effective compatibilizer to tailor a percolated network of selectively localized MWNTs and to augment the resulting conductivity. ${ }^{7}$ The study in this section will shed light on the local morphology and the associated dynamics, based on the interfacial capacitance pertaining to the MWNTs-P $\alpha$ MSAN interface in blends with various copolymers. To shift the interfacial peak fully into the measurement frequency window to further increase the accuracy of the 10 analysis, we study the interfacial polarization effects in the AC conductivity spectrum at $220 \quad 11$ 
${ }^{\circ} \mathrm{C}$. Thus, to identify the various polarization effects in the biphasic blends, the steady state AC conductivity spectrum for the blend with $0.5 \mathrm{wt} \%$ MWNTs and $0.25 \mathrm{wt} \% \mathrm{rcp}$ of 270 $\mathrm{kg} / \mathrm{mol}$ at $220^{\circ} \mathrm{C}$ is presented in Figure $3 \mathrm{a}$ as a representative example. The AC conductivity 3 spectra of the blend components as well as P $\alpha$ MSAN/PMMA blends containing 0.5 wt\% 4 MWNTs with and without $270 \mathrm{~kg} / \mathrm{mol} \mathrm{rcp}$ at $220{ }^{\circ} \mathrm{C}$ have been discussed elsewhere. ${ }^{15}$ To de- ${ }_{5}$ convolute the various contributions to the conductivity spectrum of biphasic blends with $0.5 \quad 6$ wt\% MWNTs and $0.25 \mathrm{wt} \% \mathrm{rcp}$ of $270 \mathrm{~kg} / \mathrm{mol}$, we have used the following empirical equation describing the universal AC behavior of a percolated system to model the conductivity 8 response of the blends: ${ }^{32}$

$$
\sigma^{\prime}(\omega)=\sigma_{D C}+\frac{\omega \epsilon_{0} \Delta \epsilon(\omega \tau)^{a} \sin (\pi / 2)}{1+2(\omega \tau)^{a} \cos (\pi / 2)+(\omega \tau)^{2 a}}+B \omega^{s}
$$

where $\sigma_{d c}$ is the low frequency plateau of $\mathrm{AC}$ conductivity, $\omega$ is the angular frequency, $\Delta \epsilon \quad 10$ is the dielectric strength of interfacial polarization, $\tau$ is the characteristic relaxation time, 11 $a$ is the shape parameter characteristic of the dispersion of the peak, $B$ is a temperature 12 dependent parameter and the exponent $s$ is temperature dependent and varies between $0 \quad 13$ and 1 . In eq 3 , the first term for frequency independent conductivity is a measure of the long 14 range connectivity of the MWNTs. The second term predicts the conductivity peak from 15 the interface of entrapped P $\alpha$ MSAN between adjacent MWNTs, which occurs at intermedi- $\quad 16$ ate frequencies. The dielectric interfacial relaxation was verified to be symmetric using the 17 HN function. ${ }^{28}$ In other words, the parameter characterizing the asymmetric behavior of the $\quad 18$ relaxation, $b$ in eq 1 is equal to 1 , and we have used a Cole-Cole distribution (second term 19 in eq 3) to predict the interfacial conductivity response of the biphasic blends. The third 20 power law term occurring at high frequencies describes the frequency behavior of electron 21 conduction in disordered materials (MWNTs). In Figure 3a, above $1 \mathrm{MHz}$, the short-time 22 dynamics is characteristic of hopping of MWNTs charges to a nearby spatially separated 23 localized state in which the MWNTs charges sub-diffuse through the barrier imposed by ${ }_{24}$ 
their structural disorder. ${ }^{33}$ This leads to a dispersive conductivity at high frequencies, which increases with frequency with a power law exponent ' $\mathrm{s}$ ' $\approx 0.8$. The Dyre model ${ }^{34}$ for percola- 2 tion conduction of electron-conducting disordered materials predicts $s$ between 0.7 and 1 for hopping of charge carriers over spatially random barriers. The estimated $s$ falls in the range of hopping transport between the MWNTs, ${ }^{32}$ which is schematically depicted in Figure $3 \mathrm{a} . \quad 5$ Schematics of the morphology for the various contributions to the conductivity spectrum are 6 shown in Figure 3a and the cumulative result of the fitting using eq 3 is shown as a bold line 7 in Figure 3a.

The conductivity spectrum in Figure 3 a shows the presence of an interfacial polarization at intermediate frequencies, which is the same polarization as that in Figure 1b, but shifted to higher frequencies due to the increased temperature. We will employ the permittivity spectrum to obtain further insight in the various polarizations in the studied blend system, 12 which we believe may be linked to the connectivity at various length scales, corresponding 13 to the 3 regions indicated in Figure 3a. Thereto, the permittivity spectrum of a biphasic ${ }_{14}$ blend containing $0.5 \mathrm{wt} \%$ MWNTs and $0.25 \mathrm{wt} \% \mathrm{rcp}$ of $270 \mathrm{~kg} / \mathrm{mol}$ at $220{ }^{\circ} \mathrm{C}$ is shown in ${ }_{15}$ Figure 3b. The $\epsilon^{\prime}$ above $1 \mathrm{MHz}$ is frequency independent in Figure 3b. This is due to the fact ${ }_{16}$ that the structural $(\alpha)$ and molecular $(\beta)$ relaxations of the components are outside the mea- $\quad{ }_{17}$ suring frequency window as the probed temperature of $220^{\circ} \mathrm{C}$ is well above the constituents $\mathrm{T}_{g}\left(\approx 100{ }^{\circ} \mathrm{C}\right)$. This corresponds to the region of MWNT-MWNT electron hopping in the 19 conductivity spectrum. From few $\mathrm{kHz}$ to $1 \mathrm{MHz}$, a Cole-Cole distribution for the interfacial 20 polarization in the conductivity response (Figure 3a) manifested in a step increase in $\epsilon^{\prime}$ and $\quad 21$ a corresponding Debye-like relaxation peak in $\epsilon^{\prime \prime}$ in Figure 3b. A pronounced increase in $\epsilon^{\prime} \quad 22$ and $\epsilon_{\text {conduction free }}$ is obtained below $10 \mathrm{~Hz}$ owing to the contribution from electrode polar- ${ }^{23}$ ization. As the slope of $\epsilon_{\text {conduction free }}$ is slightly less than 1 for electrode polarization, with ${ }_{24}$ decreasing frequency, $\sigma^{\prime}$ slightly decreases (using $\sigma^{\prime}=\omega \epsilon_{0} \epsilon^{\prime \prime}$ ) from the dc plateau owing to 25 the contribution of the conductivity through the imperfectly blocking double layer at the 26 electrodes. Nevertheless, the conductivity of the percolated MWNT network dominates the ${ }_{27}$ 

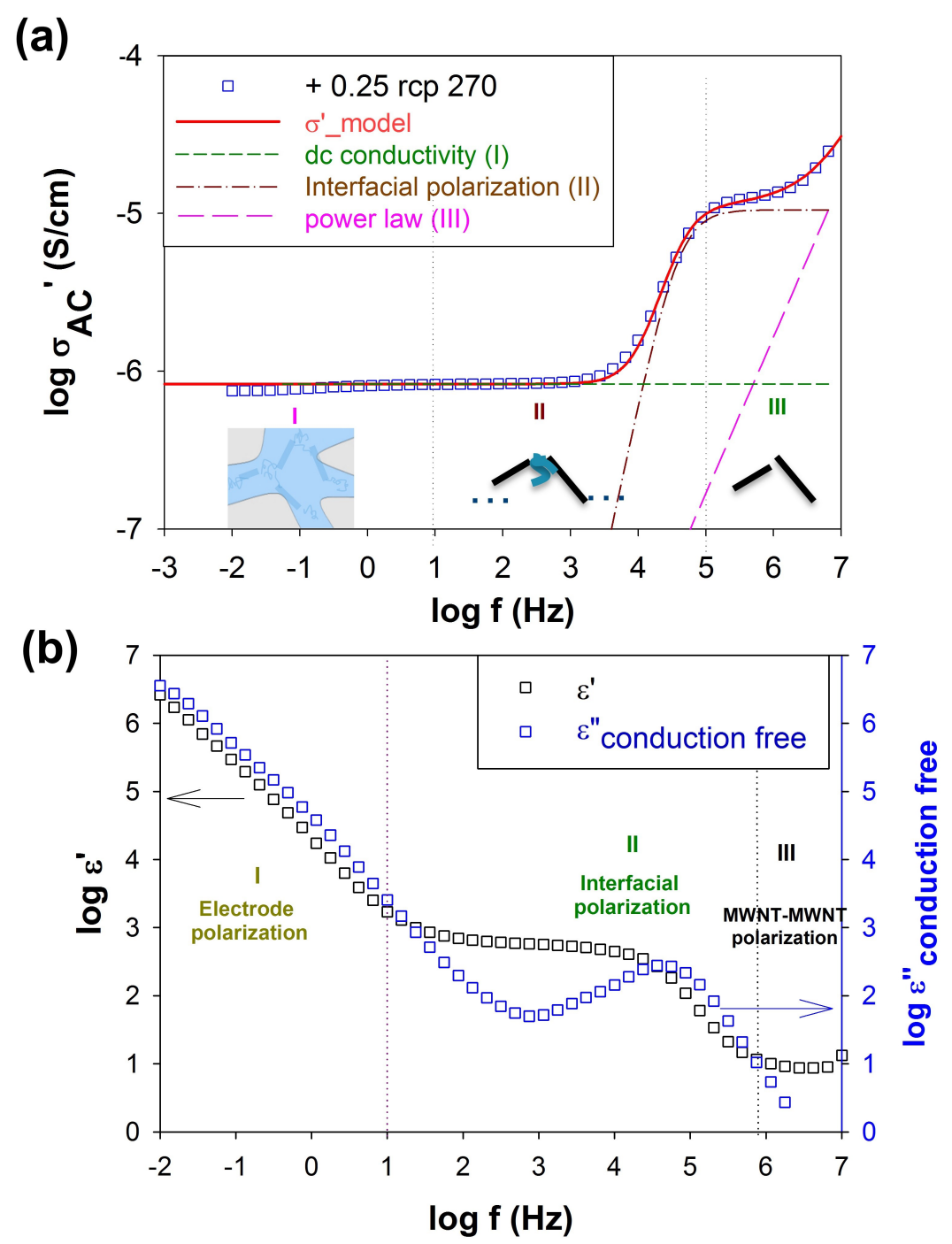

Figure 3. (a) Steady state $\mathrm{AC}$ conductivity spectrum at $220{ }^{\circ} \mathrm{C}$ for biphasic $40 / 60$ P $\alpha$ MSAN/PMMA blends with $0.5 \mathrm{wt} \%$ MWNTs and $0.25 \mathrm{wt} \% \mathrm{rcp}$ of $270 \mathrm{~kg} / \mathrm{mol}$. The fitted data (eq 3 ) is shown as red bold line. The contributions obtained from DC conductivity (term 1 in eq 3), interfacial polarization (term 2 in eq 3) and power law (term 3 in eq 3) have been demarcated. Inset: Schematic of the morphology for the various contributions of the conductivity spectrum, where MWNTs are represented by black solid lines. (b) Dielectric permittivity and "conduction free" dielectric loss spectrum at $220{ }^{\circ} \mathrm{C}$ of the biphasic blend whose steady state AC conductivity spectrum is shown in (a). The contributions from electrode polarization, entrapped $\mathrm{P} \alpha \mathrm{MSAN}-\mathrm{MWNT}$ interfacial polarization and MWNT-MWNT polarization have been demarcated.

DC conductivity as compared to the conductivity at the electrodes in Figure 3a. It should 1 be noted that the interfacial polarization in the P $\alpha$ MSAN/PMMA blends without MWNTs 2 
occurs at a frequency around $1 \mathrm{kHz}$ (data not shown) at this temperature. Due to its low dielectric strength with respect to that of the P $\alpha$ MSAN/MWNT interface, it does not show 2 up in the blends with MWNTs. In conclusion, we successfully de-convoluted the dynamic 3 response of the conductivity and permittivity spectrum of the blend. Having elucidated the various polarization contributions, the task of deconvoluting and estimating the capacitance from the various polarizations is performed by using the impedance response of the studied biphasic blend system. The equation that relates the impedance $\left(Z^{*}\right)$ with the dielectric properties is given by: ${ }^{12}$

$$
\epsilon^{*}(\omega)=\frac{1}{\iota \omega Z^{*}(\omega) C_{0}}
$$

Henceforth, the Nyquist impedance spectrum corresponding to the permittivity spectrum 9 presented in Figure 3b is shown in Figure 4a. The various polarizations manifest themselves 10 as semi-circular arcs, whose contributions as discussed in Figure 3a, b have been assigned 11 in Figure 4a. It can be seen from Figure $4 \mathrm{a}$ that the Nyquist impedance spectrum is 12 dominated by the response of the interface, which makes it an excellent tool to study this 13 process. To quantify the physical processes in the blend whose dynamics is originating 14 from local length scales, interface and surface effects, it is imperative to design an electrical 15 circuit model which mimics the response of the current through a path offering the least ${ }_{16}$ resistance to the various polarizations. ${ }^{35}$ The equivalent circuit model treats a dynamic ${ }_{17}$ process as a random mixture of resistors and capacitors. Subsequently, these elements 18 should be correlated with the physical state of the various processes which the system 19 undergoes, as shown in Figure 3a. At high frequencies, the electronic charge carriers of 20 the MWNTs scan distances between the MWNTs, whose polarization is simulated using a ${ }_{21}$ parallel combination of $R_{M W N T}$ and $C_{M W N T}$. Furthermore, the entrapment of charge at 22 the MWNT-P $\alpha$ MSAN interface with disparate properties is accounted for by another RC ${ }_{23}$ element. However, instead of an ideal capacitor, a constant phase element $\left(C P E_{\text {interface }}{ }^{24}\right.$ 


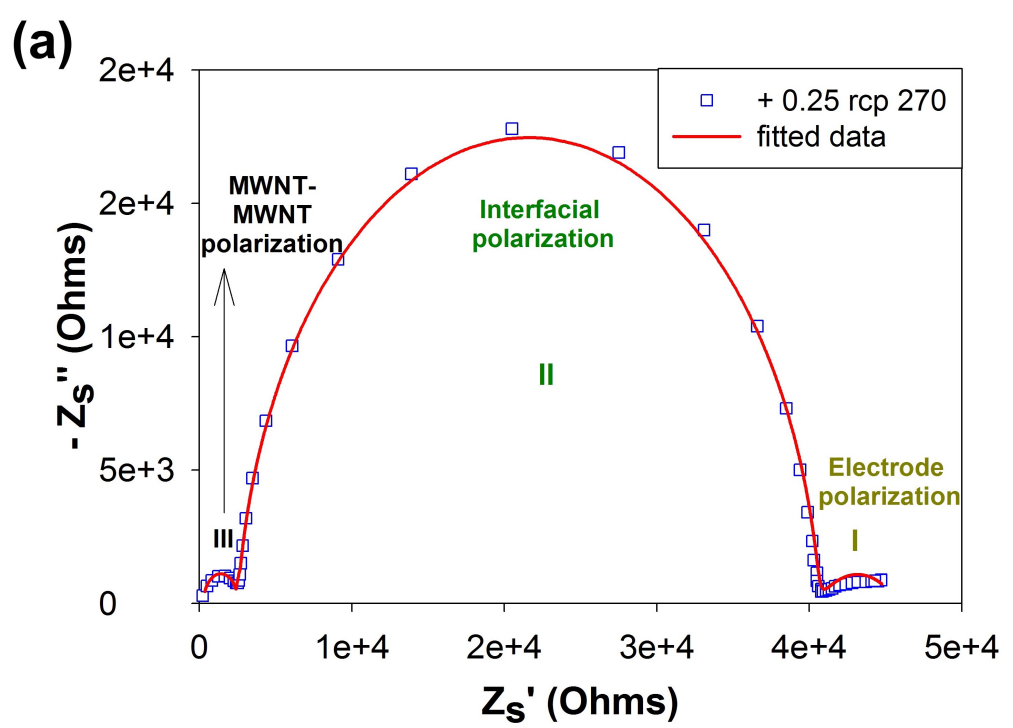

(b)

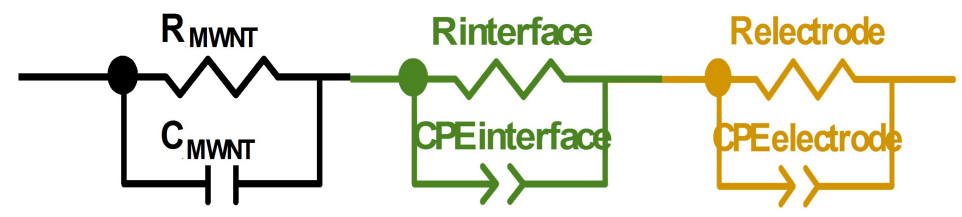

Figure 4. (a) Corresponding Nyquist impedance spectrum of the blend whose conductivity and permittivity spectra are shown in Figure $3 \mathrm{a}$ and $\mathrm{b}$, respectively. The fitted data is shown as red line using the (b) equivalent circuit consisting of a RC and 2 RQ elements in series, where CPE in the RQ element stands for constant phase element.

has been chosen to take into account the spatial gradient of the properties at the interface. 1 The resistance offered by the imperfect blocking of charges at the MWNT-P $\alpha$ MSAN 2 interface is captured by $R_{\text {interface }}$, which forms a parallel pathway with $C P E_{\text {interface }}$. The 3 impedance of the CPE is represented by $\mathrm{Z}_{C P E}^{*}=1 /\left(\mathrm{Q} \omega^{\alpha}\right)$, where $\mathrm{Q}$ takes into account the interfacial capacitance $\left(C_{\text {interface }}\right)$ and $\alpha$ denotes the frequency dependence of the 5 capacitive behavior. ${ }^{35}$ For a parallel combination of a resistor and a CPE, the $C_{\text {interface }} 6$ can be calculated from $\mathrm{Q}$ using $C_{\text {interface }}=\mathrm{Q} \omega_{\max }^{\alpha-1}$, where $\omega_{\max }$ is the frequency at which 7 Z" attains a maximum. ${ }^{36}$ Furthermore, the resistance owing to the leakage of charges at 8 the blocking electrodes $\left(R_{\text {electrode }}\right)$ forms a parallel network with the imperfect double-layer 9 capacitance $\left(C P E_{\text {electrode }}\right)$ at the electrodes. Hence, in conclusion, the impedance response 10 
of the blend was simulated with a series combination of 2 parallel RQ elements and a 1 parallel $\mathrm{RC}$ element and the result is shown in bold lines in Figure 4a. The values of the 2 $\mathrm{R}_{M W N T}$ between MWNTs for $0.5 \mathrm{wt} \%$ concentration $(\approx 10 \mathrm{kOhms})$ obtained from fitting is 3 of the same order of magnitude as the contact resistance for MWNTs undergoing hopping 4 charge transport when embedded in vulcanized silicone rubber. ${ }^{37}$ A low contact resistance 5 between the MWNTs as obtained in this study is encountered in carbon nanotubes when 6 their electronic states are coupled, ${ }^{38}$ and even between two metals with good contact. ${ }^{39}$ 7 We thus successfully simulated the experimental impedance response for all blends using 8 our developed equivalent circuit model. The physical state of the probed interface at the 9 mesoscopic length scales will be deduced in the next section via the interfacial capacitance. 10

\subsubsection{Effect of Copolymers on the Interfacial Capacitance and Resistance}

In this section, the effects of rcp and bcp molecular weight and concentration on the storage 13 ability of the MWNT charges by the entrapped P $\alpha$ MSAN between the adjacent MWNTs will ${ }_{14}$ be investigated, and will subsequently be linked to the connectivity of the entrapped poly- 15 mer - MWNTs. The charge storage is quantified by the interfacial capacitance $\left(C_{\text {interface }}\right), \quad{ }_{16}$ which was estimated from the equivalent circuit (Figure 4b) fitting of the Nyquist impedance $\quad 17$ spectrum (Figure 4a). Thus, the calculated interfacial capacitance is presented in Figure $5 \quad 18$ for blends with increasing molecular weights and copolymer concentration of rcp and bcp. 19 For both rcp cases having a copolymer $\mathrm{M}_{w}$ less than the homopolymers $\mathrm{M}_{w}$ (rcp 34 and rcp 20 192), a sluggish increase in $C_{\text {interface }}$ by a decade and saturation is observed with increasing 21 copolymer concentration. However, a significant increase in $C_{\text {interface }}$ by several decades is 22 observed for blends with only $0.25 \mathrm{wt} \%$ copolymer irrespective of the $\mathrm{M}_{w}$ of the blocks in the ${ }_{23}$ case of bcp and for rcp long enough to entangle with the homopolymers. In this regard, bcp ${ }^{24}$ of all $\mathrm{M}_{w}$ 's and long rcp improved the connectivity and refinement of the P $\alpha$ MSAN domains $\quad 25$ containing MWNTs. ${ }^{7}$ This suggests that the compatibilization of the microstructure resulted ${ }^{26}$ 


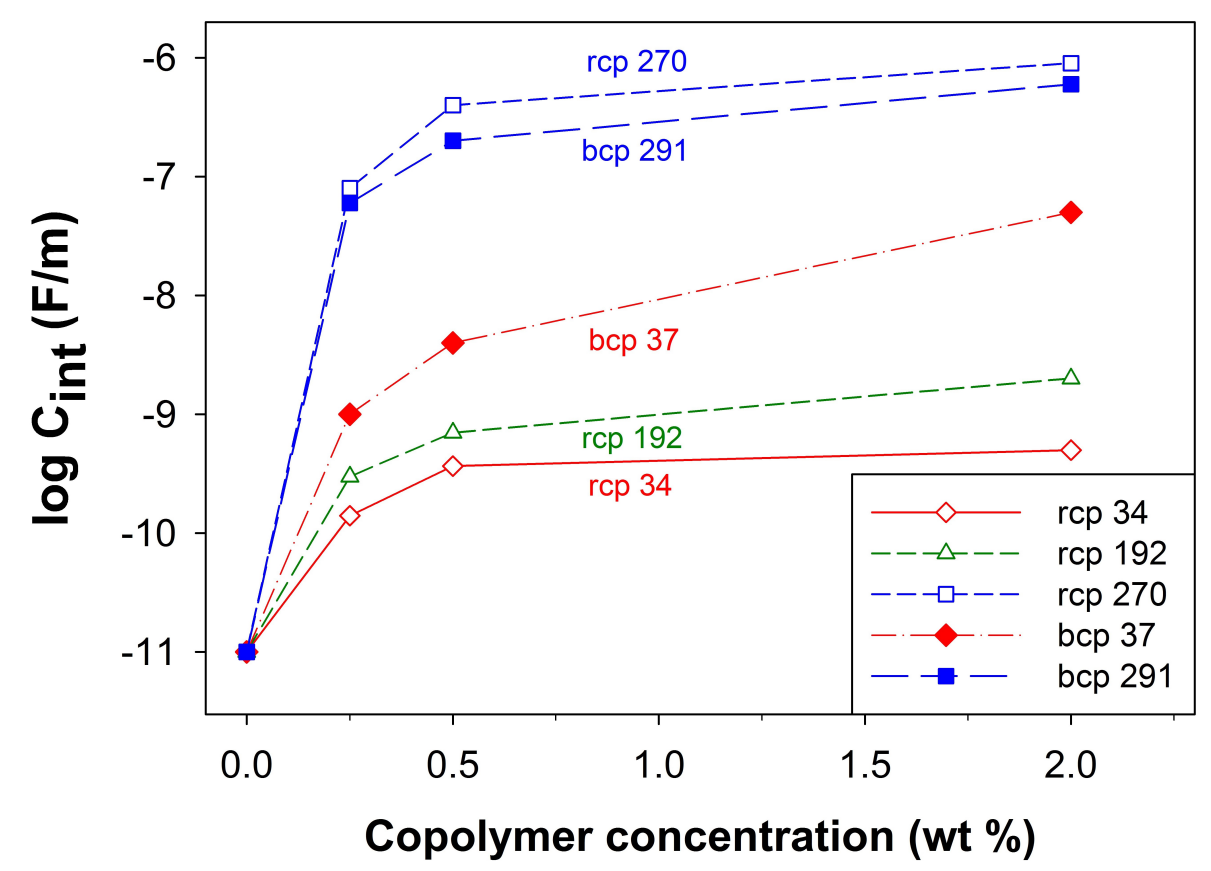

Figure 5. Interfacial capacitance of the MWNTs-entrapped P $\alpha$ MSAN network obtained from Figure 4a, b versus rcp and bcp concentration for biphasic 40/60 P $\alpha$ MSAN/PMMA blends with $0.5 \mathrm{wt} \% \mathrm{MWNTs}$ at $220{ }^{\circ} \mathrm{C}$. The number in the copolymer name indicates its molecular weight $(\mathrm{kg} / \mathrm{mol})$.

in an increase in the interfacial capacitance of the MWNTs with entrapped P $\alpha$ MSAN. The 1 trends in $C_{\text {interface }}$ for blends with various $\mathrm{rcp} / \mathrm{bcp}$ are qualitatively the same as that for the 2 electrical conductivity achieved upon increasing the long-range connectivity of the MWNT 3 network selectively localized in the P $\alpha \mathrm{MSAN}$ phase. ${ }^{7}$ Moreover, the observed increase and $\quad 4$ saturation behavior of the interfacial capacitance corresponds to a simultaneous drop and 5 saturation of the interfacial resistance $\left(R_{\text {interface }}\right)$ of the probed interface with effective com- 6 patibilization (Figure 6). In summary, effective compatibilization leads to a pronounced 7 increase in the charge storage capacity $\left(C_{\text {interface }}\right)$ of the interface formed by entrapped 8 $\mathrm{P} \alpha \mathrm{MSAN}$ between adjacent MWNTs.

The physical state and connectivity of the MWNT-P $\alpha$ MSAN interfaces exhibiting a pro- 10 nounced increase in $C_{\text {interface }}$ with increasing copolymer concentration and $\mathrm{M}_{w}$ can be un- ${ }_{11}$ derstood in terms of charge carrier diffusion in the resistor - capacitor network of entrapped 12 


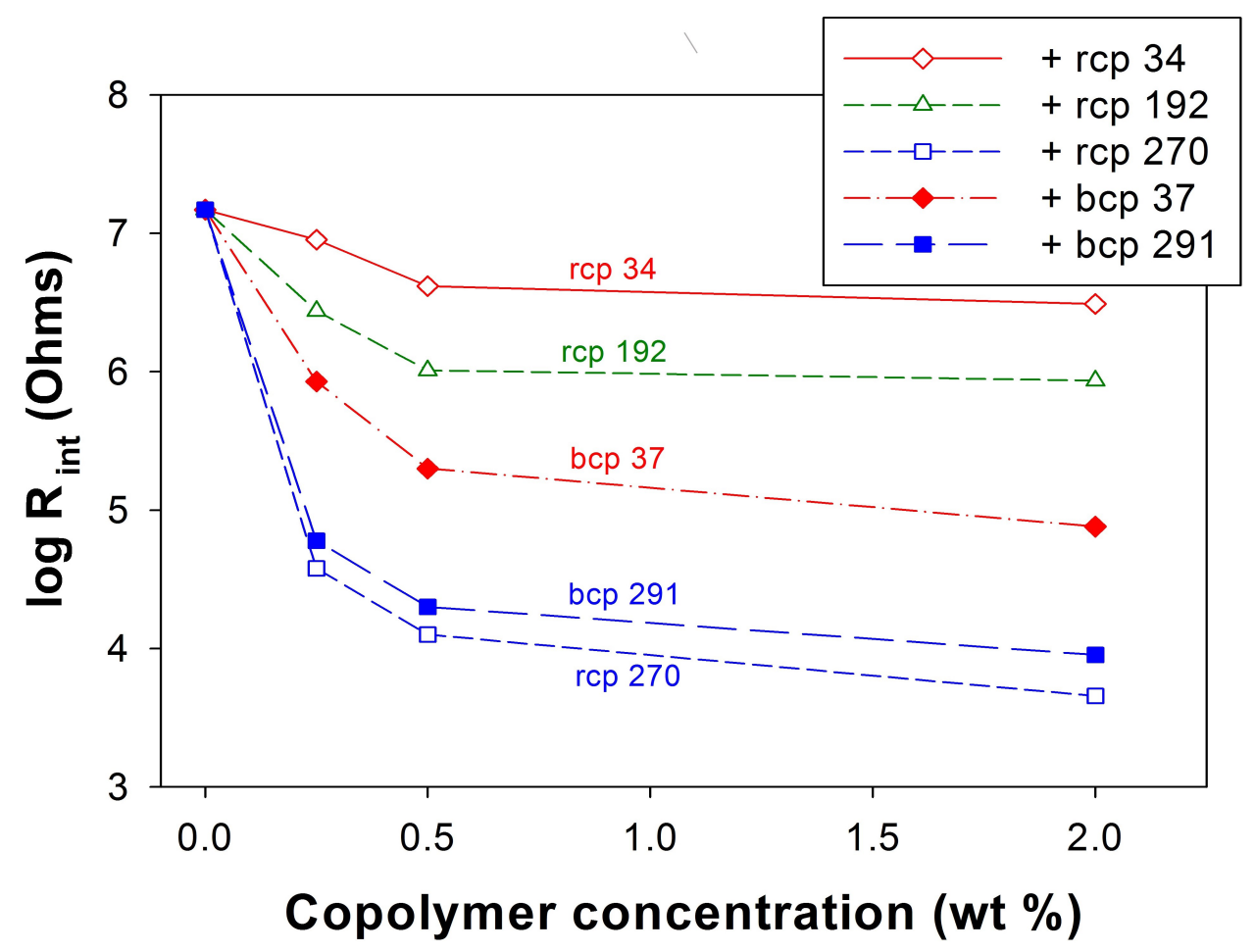

Figure 6. Interfacial resistance of the MWNTs-entrapped $\mathrm{P} \alpha \mathrm{MSAN}$ network obtained from Figure 4a, b versus rcp and bcp concentration for biphasic 40/60 P $\alpha$ MSAN/PMMA blends with $0.5 \mathrm{wt} \% \mathrm{MWNTs}$ at $220^{\circ} \mathrm{C}$. The number in the copolymer name indicates its molecular weight $(\mathrm{kg} / \mathrm{mol})$.

P $\alpha$ MSAN - MWNTs. Presence of strong polymer-filler interactions leads to entrapment of 1 the polymer chains, in which the thickness of bound polymers determines the mode and 2 dynamics of the charge carriers between the fillers. ${ }^{40}$ In the framework of the microcapacitor 3 model, two neighboring MWNTs form two electrodes enclosing a thin layer of dielectric material, and a network of these local capacitors expands between the imposed electrodes. ${ }^{41}$ Due $\quad 5$ to the resistive contribution of the entrapped P $\alpha \mathrm{MSAN}$ chains to the transport of electronic 6 charge carriers between the adjacent MWNTs, the biphasic blends do not exhibit the intrin- 7 sic conductivity $(\approx$ few $\mathrm{S} / \mathrm{cm}$ ) of MWNTs. An increase in the static permittivity (normal- 8 ized $C_{\text {interface }}$ ) with increasing filler concentration until the percolation threshold, followed 9 by saturation above the percolation threshold of the filler was reported for Polycarbonate 10 (PC)-MWNT composites. ${ }^{42}$ The behavior was attributed to the formation of microcapacitors ${ }_{11}$ 
at the gaps (and possibly free ends) between the MWNTs and the bound polymer. In our framework, increasing the continuity of the MWNT-containing P $\alpha$ MSAN phase by means of 2 copolymers leads to the formation of an extended continuous network of microcapacitors. In summary, the pronounced effect of increased connectivity of MWNT-entrapped P $\alpha$ MSAN on its capacitive properties was successfully demonstrated in this section.

\subsection{Gap spacing of the MWNT-P $\alpha$ MSAN microcapacitor}

The physical state of the entrapped polymer between the fillers dictates the stress transfer 7 by the filler network, and the characteristic gaps between the MWNTs engender a barrier for the transportation of charge through the entire filler network. ${ }^{43}$ Hence, the physical state and characteristic gaps of the probed MWNT-P $\alpha$ MSAN elements in our biphasic blend system are relevant for the properties and can be probed via the interfacial relaxation. An 11 estimation of the characteristic gap of the microcapacitors can be made from the interfacial 12 resistance and capacitance when knowing the mechanism of conductivity in the MWNT- ${ }_{13}$ $\mathrm{P} \alpha \mathrm{MSAN}$ elements forming the microcapacitors. The presence of the dielectric P $\alpha \mathrm{MSAN}{ }_{14}$ between neighboring conducting MWNTs results in a potential barrier to inter-nanotube 15 hopping. This can result in a different conduction mechanism, whose barrier and tempera- ${ }_{16}$ ture dependence of conductivity could be different from that for hopping of MWNT charge ${ }_{17}$ carriers. Based on the fact that percolating P $\alpha$ MSAN nanocomposites and biphasic blends ${ }_{18}$ with MWNTs do not exhibit the intrinsic conductivity of MWNTs $(\approx$ few S/cm), we surmise $\quad 19$ that the electron transport through the P $\alpha$ MSAN between adjacent MWNTs is controlled 20 by fluctuation induced tunneling, as reported by Kilbride et al. ${ }^{44}$ for PmPV and PVA based ${ }_{21}$ carbon nanotube composites and Fritzsche et al. ${ }^{43}$ for carbon black-SB rubber composites. $\quad 22$ Since the temperature dependence of the conductivity contains intrinsic information about ${ }^{23}$ the imposed barrier to electron transfer and the mechanism of conductivity, the logarithm of ${ }_{24}^{24}$ the DC conductivity of the MWNT-P $\alpha$ MSAN nanocomposite having equivalent concentra- ${ }_{25}$ tion of MWNTs $(1.25 \mathrm{wt} \%)$ as in the blend $(0.5 \mathrm{wt} \%)$ was plotted versus the reciprocal of the $\quad 26$ 
absolute temperature. A strong linear dependence was obtained indicative of the presence of thermal fluctuation induced tunneling (FIT). ${ }^{17,45}$ The activation energy of the thermally activated FIT process was calculated by expressing the FIT model $\left(\sigma=\sigma_{0} \exp \left[-T_{1} /\left(\mathrm{T}+T_{0}\right)\right]\right)^{46} \quad 3$ in the form of a VFT equation (eq 2) for conductivity $\left(\sigma=\sigma_{0} \exp \left[E_{v} / \mathrm{R}\left(\mathrm{T}-T_{v}\right)\right]\right)^{29,30}$. Here, ${ }_{4}$ $\sigma_{0}$ is the pre-exponential factor, $T_{1}$ is the energy barrier imposed for the electron to tunnel 5 the insulating barrier and is analogous to the Vogel activation energy $\left(E_{v}\right), \mathrm{R}$ is the uni- 6 versal gas constant and $T_{0}$ is an estimate of the lowest temperature above which tunneling 7 is thermally active corresponding to the Vogel temperature $T_{v}$. Thus, the activation energy 8 was estimated to be $\approx 0.9 \mathrm{eV}$ from the slope of the temperature dependence of the $\mathrm{DC} \quad 9$ conductivity. The predicted activation energy is in reasonable agreement with the values 10 obtained for tunneling of carbon black charges across barriers of polyvinylchloride ${ }^{46}$ and for ${ }_{11}$ the conductivity owing to tunneling of MWNT electrons through the barriers imposed by 12 the adsorbed PVDF near and above the $T_{g}$ of PVDF. ${ }^{17}$ In this respect, the fitting param- ${ }_{13}$ eters and the activation energy obtained from the FIT model depend on the interaction of 14 the polymer matrix and the filler, the aspect ratio and the concentration of the filler. ${ }^{47}$ In ${ }^{15}$ summary, at high frequencies the electrons hop between the neighboring MWNTs, whereas 16 at intermediate frequencies, the electrons of MWNTs tunnel through the barriers imposed ${ }_{17}$ by the entrapped P $\alpha$ MSAN. 18 The interfacial relaxation process is attributed to the transfer of electrons of MWNTs by tun- ${ }_{19}$ neling through the gap occupied by the entrapped polymer between the adjacent MWNTs. 20 In this respect, the gap between the adjacent filler particles determines the barrier, and ${ }_{21}$ hence, the interfacial resistance and capacitance between the adjacent fillers. In the case of 22 fluctuation induced tunneling, the capacitance shows the typical inverse relationship with the ${ }_{23}$ gap width $\delta$ whereas the model predicts an exponential growth of the interfacial resistance ${ }_{24}$ with increasing gap between the MWNTs, given by: ${ }^{48} 25$

$$
C_{\text {interface }}=\epsilon_{0} \epsilon \frac{A}{\delta}
$$




$$
\begin{aligned}
R_{\text {interface }} & =\frac{16 \pi^{2} \hbar}{3 e^{2}} \frac{\delta}{k_{0} A} \exp \left(k_{0} \delta\right) \\
k_{0} & =\frac{2 \sqrt{2 m_{e}}}{\hbar} \sqrt{V}
\end{aligned}
$$

where $A$ is the cross section of the capacitor, $e$ the electronic charge, $m_{e}$ the mass of an 2 electron, $\hbar$ the Planck's constant and $k_{0}$ depends on the height of the potential barrier 3 $V$. The combined effects of the capacitor width as well as area in eqs. $5-7$ complicate 4 determination of any of these parameters from $R_{\text {interface }}$ and $C_{\text {interface }}$. However, from the 5 relaxation time for the tunneling process $\left(\tau_{\text {interface }}\right)$ obtained by combining $R_{\text {interface }}$ and 6 $C_{\text {interface, }}$ the gap spacing can be obtained: ${ }^{43}$

$$
1 / \tau_{\text {interface }}=1 / R_{\text {interface }} C_{\text {interface }}=\frac{3 e^{2}}{16 \pi^{2} \hbar} \frac{k_{0}}{\epsilon} \exp \left(-k_{0} \delta\right)
$$

As an alternative to the combination of $R_{\text {interface }}$ and $C_{\text {interface }}, \tau_{\text {interface }}$ can also be obtained $\quad 8$ directly from the HN fitting of the interfacial relaxation in the "conduction free" dielectric 9 loss spectra (Figure 3b). Both approaches exhibited a remarkable agreement, which serves 10 as an indirect proof of the accuracy of the estimated $R_{\text {interface }}$ and $C_{\text {interface }}$ values from ${ }_{11}$ Figure 4 a.

As the capacitance scales inversely with the thickness of the insulator region (eq 5), ${ }^{12}$ a ${ }^{13}$ smaller gap would lead to a decrease in the contact resistance and an increase in the interfacial 14 capacitance. The dielectric constant of $\mathrm{P} \alpha \mathrm{MSAN}$ in the capacitor for estimating the gaps in 15 eq 8 was taken to be 3.1. For a potential barrier of $0.5 \mathrm{eV}$, characteristic for MWNTs, ${ }^{18,33}{ }_{16}$ the gap of the capacitor was found to be $\approx 21 \mathrm{~nm}$ for blends with only $0.5 \mathrm{wt} \%$ MWNTs. $\quad 17$ Furthermore, the gap spacing was estimated to be 15 - $18 \mathrm{~nm}$ for blends with $0.5 \mathrm{wt} \% \quad 18$ MWNTs and with increasing concentrations of various rcp and bcp. The invariant gap 19 spacing with copolymer architecture and concentration is expected as we have proven via 20 rheology and conductivity measurements that the copolymer does not affect the dispersion 21 of the MWNTs in P $\alpha$ MSAN-MWNT nanocomposites. ${ }^{15}$ As a comparison, values of 11 and 22 $8 \mathrm{~nm}$ for the gap of capacitors of SBR between carbon black aggregates were obtained ${ }_{23}$ 
for low carbon black concentrations $(30 \mathrm{phr})$ for a barrier potential of $0.1 \mathrm{eV}$ and $0.2 \mathrm{eV}, \quad 1$ respectively. ${ }^{43}$ In summary, we successfully estimated the gap spacing of the microcapacitors 2 formed by the entrapped P $\alpha$ MSAN between adjacent MWNTs and its invariant behavior 3 with the copolymer from both dielectric relaxation as well as the impedance formalism. $\quad 4$

\subsection{Amount of entrapped P $\alpha \mathrm{MSAN}$}

As the gap spacing of the microcapacitors is unaffected by the copolymer, only the con- 6 nectivity of the microcapacitors could tune the interfacial capacitance of the microcapacitor 7 network. To gain further insight in the effect of various copolymers on the connectivity of the 8 microcapacitors, the physical parameters characterizing the microcapacitor assembly will be 9 estimated. Thereto, we employed the theoretical interlayer model developed by Steeman et 10 al. ${ }^{49}$ The model describes the complex permittivity of a three-phase blend $\left(\epsilon_{b}^{*}\right)$ comprising of 11 filler, interlayer and matrix, in which filler particles enclosed with a layer of interfacial mate- 12 rial are embedded in a matrix. The response of our blend system to the imposed electric field 13 allows to allocate the various blend components to the relevant constituents of the model. ${ }_{14}$ The electric field response of entrapped P $\alpha$ MSAN chains between the MWNTs results in 15 a substantial capacitance and thus, the former behaves as a material with high dielectric 16 constant. This phenomenon is due to the partial screening of the entrapped P $\alpha$ MSAN (be- ${ }_{17}$ having as fillers) by the enveloped interlayer of conductive MWNTs embedded in the blend. ${ }_{18}$ A similar study has been performed by Steeman et al. for model composites of interfacially 19 adsorbed water on spherical glass filler particles dispersed in a matrix of HDPE in order to 20 estimate the amount of adsorbed water. ${ }^{50,51}$ The modelling of $\epsilon_{b}^{*}(\omega)$ is achieved by taking 21 into account the respective complex permittivity and volume fractions of the filler $\left(\epsilon_{f}^{*}(\omega) \quad 22\right.$ and $\phi_{f}$ of $\left.\mathrm{P} \alpha \mathrm{MSAN}\right)$, the interlayer $\left(\epsilon_{l}^{*}(\omega)\right.$ and $\phi_{l}$ of MWNTs $)$ and the matrix $\left(\epsilon_{m}^{*}(\omega)\right.$ and ${ }_{23}$ $\phi_{m}$ of the blend with copolymer only). For the interlayer, $\phi_{l}$ was assumed to be constant ${ }_{24}$ (MWNT volume fraction in the blend) and the $\epsilon_{l}^{*}(\omega)$ was calculated using eq 9 in which the 25 
capacitive contribution of the MWNTs was neglected $\left(\epsilon_{l}^{\prime}(\omega) \approx 0\right)$ :

$$
\epsilon_{l}^{*}(\omega)=i \epsilon_{l}^{\prime \prime}(\omega)=i \frac{\sigma_{d c}^{\prime} \omega}{\epsilon_{0}}
$$

The $\sigma_{d c}^{\prime}$ for MWNTs used in eq 9 is $10^{4} \mathrm{~S} / \mathrm{cm} .{ }^{27}$ Subsequently, $\epsilon_{b}^{*}$ can be estimated in 2 the direction of any of the principal axes of the filler by incorporating the shape factor of 3 the filler for that direction, and subsequently weighing the contributions with their relative occurrence. In this view, an experimental Debye-type dielectric interfacial peak observed in Figure $3 \mathrm{~b}$ is a manifestation of a negligible distribution in filler dimensions and shape 6 factors, ${ }^{49}$ allowing to choose the appropriate form of the interlayer model: ${ }^{49}$

$$
\begin{gathered}
\epsilon_{b}^{*}(\omega)=\frac{\epsilon_{f}^{*}(\omega) \phi_{f}+\epsilon_{l}^{*}(\omega) \phi_{l} R+\epsilon_{m}^{*}(\omega) \phi_{m} S}{\phi_{f}+\phi_{l} R+\phi_{m} S} \\
\mathrm{R}=\frac{(1-n) \epsilon_{l}^{*}(\omega)+n \epsilon_{f}^{*}(\omega)}{\epsilon_{l}^{*}(\omega)} \\
\mathrm{S}=\frac{\left(n \epsilon_{l}^{*}(\omega)+(1-n) \epsilon_{m}^{*}(\omega)\right)\left(n \epsilon_{f}^{*}(\omega)+(1-n) \epsilon_{l}^{*}(\omega)\right)+d n(1-n)\left(\epsilon_{l}^{*}(\omega)-\epsilon_{m}^{*}(\omega)\right)\left(\epsilon_{f}^{*}(\omega)-\epsilon_{l}^{*}(\omega)\right)}{\epsilon_{l}^{*}(\omega) \epsilon_{m}^{*}(\omega)} \\
\mathrm{d}=\frac{\phi_{f}}{\phi_{f}+\phi_{l}}
\end{gathered}
$$

During the estimation of $\epsilon_{b}^{*}$, the first fitting parameter is $n$, which characterizes the shape 8 of the polarized P $\alpha$ MSAN entrapped between the MWNTs, and is a measure of the in- 9 terfacial polarization strength of a single microcapacitor. The other fitting parameter is 10 the total volume fraction of the polarized $\mathrm{P} \alpha \mathrm{MSAN}\left(\phi_{f}\right)$ enveloped by the MWNTs in the 11 blend, which is correlated with the connectivity of the microcapacitors. In summary, from 12 the interlayer model, the combined knowledge of the physical state and connectivity of the 13 microcapacitors estimated respectively from the shape factor of P $\alpha \mathrm{MSAN}$ in a single micro- ${ }_{14}$ capacitor and the volume fraction of the entrapped P $\alpha$ MSAN in the blend determine the 15 total dielectric interfacial polarization of the microcapacitors in the blends. In this regard, 16 the various copolymers ability to generate and stabilize the biphasic morphology in order 17 to pave a network of microcapacitors will be predicted by the combined knowledge of the 18 
(a)
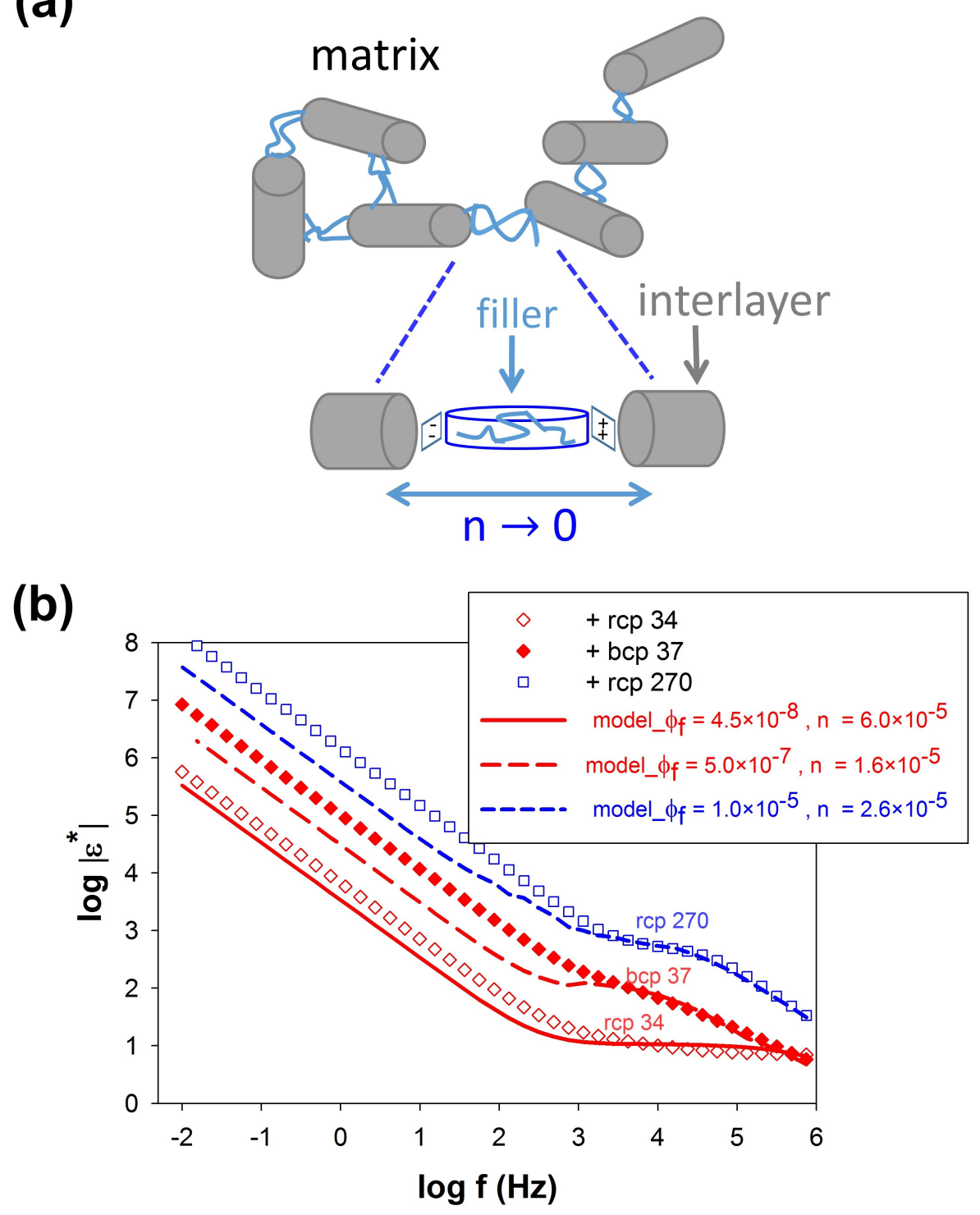

Figure 7. (a) Schematic of the proposed arrangement of the various components in the interlayer model used for modelling the complex permittivity spectra for blends with MWNTs and copolymer. (b) Complex permittivity spectra of biphasic 40/60 P $\alpha$ MSAN/PMMA blend with $0.5 \mathrm{wt} \%$ MWNTs and $0.25 \mathrm{wt} \%$ short rcp $(34 \mathrm{~kg} / \mathrm{mol})$, long rcp $(270 \mathrm{~kg} / \mathrm{mol})$ and short bcp $(37 \mathrm{~kg} / \mathrm{mol})$ at $220{ }^{\circ} \mathrm{C}$. The fitted spectra obtained using the interlayer model (eq 10) are shown as lines and the values of the fitting parameters are indicated.

microcapacitor behavior and the interlayer model.

We analyze the interfacial dielectric response of entrapped P $\alpha$ MSAN filler sandwitched be- 2 tween two neighboring MWNTs mimicking an interlayer, whose possible arrangements are 
depicted in the schematic presented in Figure 7a. As a proof of concept, Figure 7b shows the 1 complex dielectric permittivity spectrum of a biphasic blend with $0.25 \mathrm{wt} \%$ long rcp $(270 \quad 2$ $\mathrm{kg} / \mathrm{mol}$ ) and $0.5 \mathrm{wt} \% \mathrm{MWNTs}$ at $220^{\circ} \mathrm{C}$, whose permittivity and impedance response are 3 presented in Figure 3b and Figure 4a. For comparison, the spectra of blends with short rep 4 and short bcp are also shown. An accurate prediction of the contribution from interfacial 5 polarization is obtained for the various blends, as shown in Figure $7 \mathrm{~b}$ (lines). The fit at 6 low frequencies is not accurate due to contributions of electrode polarization, which are not 7 taken into account in the model. The fitting parameter ' $n$ ' is estimated to be $\approx 10^{-5}$ and is 8 unaffected by the copolymer type and concentration. This further warrants that the copoly- 9 mer does not affect the state of dispersion of the MWNTs. A comment on the physical 10 state and the plausible network formed by the entrapped P $\alpha$ MSAN between neighboring ${ }_{11}$ MWNTs is done. With respect to the former, fillers with ' $n$ ' $\rightarrow 0$ are predicted to behave ${ }_{12}$ rod-like along the direction of the electric field. ${ }^{49}$ This is in agreement with the fact that ${ }_{13}$ the gap spacing of the P $\alpha \mathrm{MSAN}$ microcapacitor is larger than the thickness of the polarized ${ }_{14}$ $\mathrm{P} \alpha \mathrm{MSAN}$ region forming the microcapacitor. The latter can be understood by the fact that 15 the microcapacitor is characterized by a very localized polarization zone on the MWNTs. 16 We believe the rod-like P $\alpha$ MSAN regions between adjacent MWNTs are bridging the neigh- $\quad{ }_{17}$ boring MWNTs (Figure 7a). It should be noted that ' $n$ ' $\rightarrow 0$ does not imply an anisotropy 18 in the MWNT network but rather that the dominant contributions originate from the po- ${ }_{19}$ larized P $\alpha$ MSAN microcapacitors oriented along the electric field. This was confirmed by 20 verifying that any contributions with larger ' $n$ ' values in eq 10 resulted in significantly lower ${ }_{21}$ dielectric strengths. The polymer bridges between neighboring MWNT particles determine 22 the properties of the MWNT network. Similar formation of glassy-like polymer bridges of ${ }_{23}$ styrene butadiene rubber between two carbon black aggregates were found on the basis of ${ }_{24}$ the microcapacitor model. ${ }^{43,52}$ Mainly systems with strong polymer-filler interactions exhib- ${ }^{25}$ ited formation of polymer bridges between neighboring filler particles, which resulted in a ${ }_{26}$ reduced chain mobility close to the filler surface and governed the viscoelastic response of ${ }_{27}$ 
the filler network. ${ }^{43}$

To gain further insight in the effect of various copolymers on the connectivity of the mi- 2 crocapacitors, another physical parameter linked with the connectivity, namely the volume 3 fraction of entrapped P $\alpha \mathrm{MSAN}\left(\phi_{f}\right)$ between the surfaces of MWNTs is estimated. Fig- ${ }_{4}$ ure $7 \mathrm{~b}$ shows that blends with short bcp exhibit an increase in the fitting parameter $\phi_{f}$ by ${ }_{5}$ a decade as compared to blends with short rcp, and blends with long rep show a further 6 increase in $\phi_{f}$ by more than a decade. To gain further insight in the effect of various rcp 7 and bcp on the filler connectivity, the dependence of the volume fraction of the entrapped 8 P $\alpha$ MSAN between neighboring MWNTs on the concentrations of various rcp and bcp is 9 plotted in Figure 8. The dependence of $\phi_{f}$ on the copolymer concentration exhibits a similar 10 behavior as the interfacial capacitance in the presence of various rcp and bcp (Figure 5). ${ }_{11}$ Eq 5 shows that the total capacitance area of polarized P $\alpha$ MSAN between MWNTs is pro- ${ }_{12}$ portional to the interfacial capacitance. Therefore, the total capacitance area is expected 13 to exhibit a similar behavior as the estimated $\phi_{f}$, as both physical parameters account for ${ }_{14}$ the connectivity of the microcapacitors. A pronounced increase by 4 orders of magnitude in 15 the volume fraction of the P $\alpha$ MSAN microcapacitors is in conjunction with the increasing 16 interfacial capacitance with various copolymers. The increase in the connectivity of the mi- ${ }_{17}$ crocapacitors and the interfacial capacitance of the entrapped P $\alpha$ MSAN network between $\quad 18$ neighboring MWNTs results from the ability of the copolymer to increase the refinement and $\quad 19$ to improve the connectivity of the P $\alpha \mathrm{MSAN}$ phase containing the MWNTs. This refinement 20 and improved connectivity in the presence of long rcp and bcp irrespective of its $\mathrm{M}_{w}$ was ${ }_{21}$ reported in our recent work. ${ }^{7}$ In summary, the interlayer model predicts an increase in the 22 volume fraction of entrapped rod-like $\mathrm{P} \alpha \mathrm{MSAN}$ regions by 4 decades with an increase in the ${ }_{23}$ interfacial capacitance of the MWNT-P $\alpha$ MSAN microcapacitor network. 24 


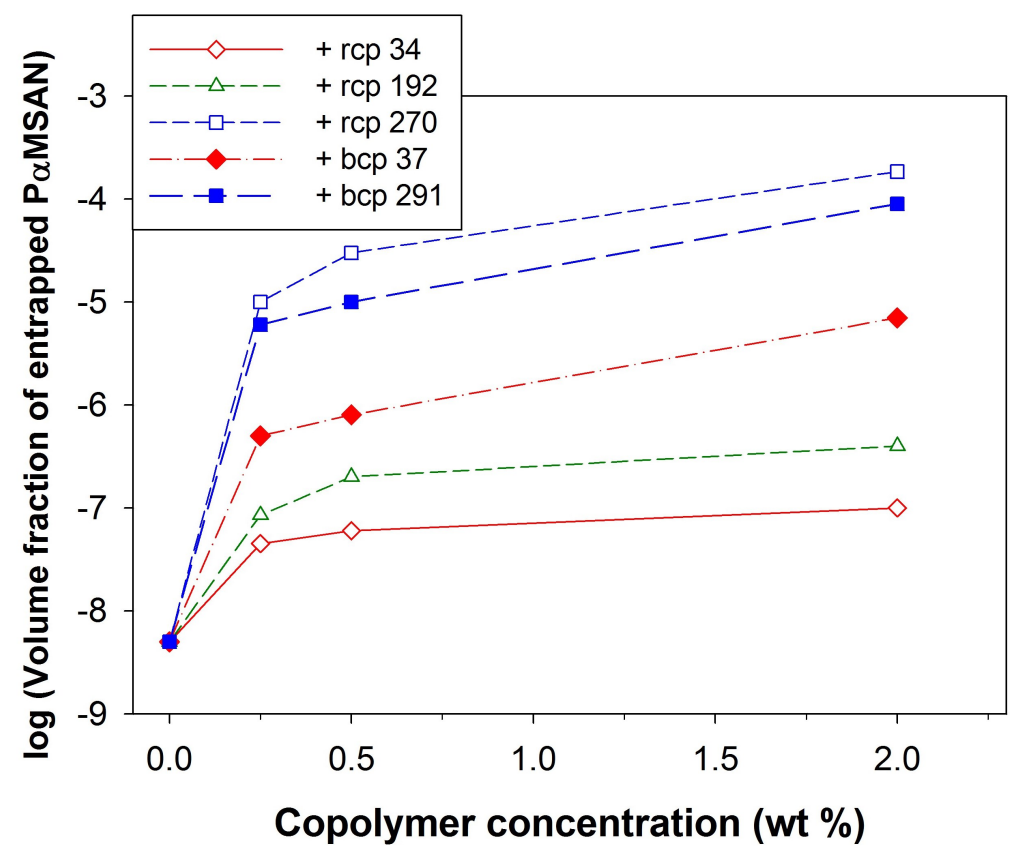

Figure 8. Volume fraction of entrapped $\mathrm{P} \alpha \mathrm{MSAN}$ obtained as a fitting parameter using eq 10 versus rcp and bcp concentration for biphasic 40/60 P $\alpha$ MSAN/PMMA blends with $0.5 \mathrm{wt} \%$ MWNTs at $220{ }^{\circ} \mathrm{C}$.

\subsection{Modeling the MWNT-P $\alpha$ MSAN interfacial capacitance}

The parameters characterizing the physical state (gap spacing) and connectivity of the entrapped P $\alpha$ MSAN-MWNTs (volume fraction of P $\alpha$ MSAN) will be used to predict the interfacial capacitance. We envisage that an estimation of the volume fraction of the entrapped polymer would incorporate the effect of the copolymer on the connectivity of the MWNTsentrapped P $\alpha$ MSAN assembly. This in turn would allow to estimate the total number of capacitors contributing to the estimated interfacial capacitance in our blend system. As the charge transfer path is contingent upon the connectivity of the MWNTs-entrapped P $\alpha$ MSAN assembly and the gap spacing is invariant with copolymer, only an increase in the connec- 9 tivity of the microcapacitors would affect the interfacial capacitance of the microcapacitor 10 assembly. The underlying motivation for the ensuing discussions is the fact that a combined 11 estimation of the total number of capacitors and the individual capacitance (from the gap 12 
spacing) would allow us to predict the total interfacial capacitance of the microcapacitor 1 assembly in the blend system. In conclusion, the estimated total interfacial capacitance is a 2 fingerprint of the connectivity of the microcapacitor assembly or the biphasic morphology, 3 whose estimated values are contingent on the copolymers architecture and concentration. $\quad 4$ As a first approximation, the product of the calculated individual capacitance $\left(C_{i}\right)$ and the 5 number of capacitors $(\mathrm{N})$ yields the total interfacial capacitance $\left(C_{\text {int }}\right)$ :

$$
C_{\text {int }}=C_{i} N
$$

The shape of each individual capacitor irrespective of the MWNTs arrangement can be 7 approximated by a cylinder, leading to:

$$
C_{i}=\epsilon_{0} \epsilon \frac{A}{\delta}
$$

with $A$ the capacitor area and $\delta$ its thickness. The ratio of the total volume of capacitors $\left(V_{t}\right)$ to the individual capacitor volume $\left(V_{i}\right)$ is used to calculate the number of capacitors 10 $(\mathrm{N})$ :

$$
N=\frac{V_{t}}{V_{i}}
$$

The product of the estimated volume fraction of $\mathrm{P} \alpha \mathrm{MSAN}$ contributing to the microcapac- $\quad{ }_{12}$ itors $\left(\phi_{f}\right)$ using the interlayer model (Figure $8 \mathrm{~b}$ ) and the blend volume is used to calculate 13 the total volume of capacitors $\left(V_{t}\right)$ :

$$
V_{t}=\phi_{f} V_{b}
$$

whereas $V_{i}$ is the volume of a cylinder:

$$
V_{i}=\delta A
$$


Upon substitution of $C_{i}$ and $\mathrm{N}$ from eq 12 - 13 in eq 11 using eq $14-15$, the $C_{i n t}$ is found 1 to be independent of the polarization area and proportional to $\phi_{f}$ :

$$
C_{i n t}=\frac{\epsilon_{0} \epsilon \phi_{f} V_{b}}{\delta^{2}}
$$

Other parameters in eq 16 are unaffected by the connectivity of the MWNT-P $\alpha$ MSAN mi- 3 crocapacitor network. It is imperative to emphasize that the total interfacial capacitance of 4 the microcapacitor assembly in eq 16 is contingent on the gap spacing estimated using the 5 FIT model and the experimental interfacial relaxation time (eq 8), and the volume fraction 6 of the microcapacitors obtained from the interlayer model and the experimental interfacial 7 relaxation strength (eq 10). The thus obtained total interfacial capacitances for blends with 8 various copolymers and with increasing copolymer concentration are presented in Figure 9.9 The interfacial capacitance obtained from the fitting of the impedance response of the blends 10 (Figure $4 \mathrm{a}$ and b) presented in Figure 5 is also shown for comparison in Figure 9. Upon ${ }_{11}$ comparison of the magnitude of the interfacial capacitance determined from the circuit fit- 12 ting of the impedance response of the blends with that estimated from the response of the 13 microcapacitor assembly in the blends using the FIT and interlayer models, a remarkable ${ }_{14}$ agreement is obtained in Figure 9. It is imperative to emphasize that the underlying ra- ${ }_{15}$ tionale in the estimation of the total capacitance has emanated from the unified formalism 16 of experimentally determined relaxation times analyzed with the FIT model in combination $\quad 17$ with experimentally determined relaxation strengths analyzed with the interlayer model. 18 Thus, the adopted approach allows to separately obtain the contributions from each capaci- 19 tor as well their connectivity and the fact that recombining the information leads to a good 20 description proofs the validity of the approach. 21 A remarkable agreement of the magnitude of the interfacial capacitance determined from the 22 circuit fitting of the impedance response of the blends with that estimated from the microca- ${ }_{23}$ pacitor assembly in the blends using various models in Figure 9 entails some discussion. Upon $\quad 24$ 
increase of the connectivity of P $\alpha$ MSAN laden with MWNTs, there are more connections of the individual microcapacitors entrapped between the surface of MWNTs, whereas the gap of each microcapacitor is not affected. Even though the charges take the least resistive path, 3 the unaltered gap of the microcapacitors in the presence of various copolymers ensures that 4 we do not affect the local charge transfer path or resistance between the microcapacitors. 5 Hence, upon increasing the refinement and continuity of the P $\alpha$ MSAN phase with copoly- 6 mers, only the effect of the connectivity of the microcapacitors orchestrates the estimated 7 interfacial capacitance. In this regard, a proportional decrease in the interfacial resistance 8 and an increase in the interfacial capacitance with increased number of capacitors is expected 9 for a parallel combination of microcapacitors $\left(C_{t o t}=\sum C_{i}=\mathrm{n} C_{i}\right.$ and $\left.R_{t o t}=\frac{1}{\sum 1 / R_{i}}=\frac{R_{i}}{n}\right) \quad 10$ and a reverse trend is expected for a series combination $\left(C_{t o t}=\frac{1}{\sum 1 / C_{i}}=\frac{C_{i}}{n}\right.$ and $R_{t o t}={ }_{11}$ $\left.\sum R_{i}=\mathrm{n} R_{i}\right)$. However, the interfacial relaxation time of the microcapacitors $\left(\tau_{\text {interface }}={ }_{12}\right.$ $\left.\frac{1}{R C}=\frac{1}{R_{i} C_{i}}\right)$ is unaffected by the choice of the charge transfer path (series or parallel). The ${ }^{13}$ latter clarifies why one single relaxation time is obtained for all systems, irrespective of the 14 complex connections of the different (but identical) micro-capacitors. Furthermore, we ob- 15 serve an increase in the interfacial capacitance and a proportional decrease in the interfacial 16 resistance with increasing amount of the copolymer (Figure 3). This suggests that the length 17 of each path for charge transfer is unaffected with refinement, but an increase in the number $\quad 18$ of connections is achieved. Thus, we are measuring the increased amount of parallel RC 19 circuits upon copolymer addition. With regard to the biphasic morphology, for disconnected 20 P $\alpha$ MSAN domains with MWNTs, the parallel contribution of the charge transfer through ${ }_{21}$ an RC network of $\mathrm{P} \alpha \mathrm{MSAN}$ and MWNTs to the interfacial capacitance is restricted. By 22 changing the copolymer type, $M_{w}$ or concentration, morphology refinement and increased ${ }_{23}$ continuity of the P $\alpha$ MSAN phase resulted in an increase in the parallel networks of the en- ${ }_{24}$ trapped P $\alpha$ MSAN microcapacitors between the surfaces of adjacent MWNTs. This leads to 25 an increase in the interfacial capacitance by around 5 decades in magnitude for percolated 26 blends as compared to blends lacking interconnectivity of the capacitors. The interlayer ${ }_{27}$ 
model also comments on the correlation of the physical state of the microcapacitors with 1 their connectivity depending upon the state of polarization of the microcapacitors. In this 2 regard, an assembly of conducting-dielectric elements with rod-like fillers tends to exhibit 3 parallel connections. ${ }^{49}$ Hence, the formation of parallel elements in the limit of rod-like filler $\quad 4$ particles of entrapped P $\alpha$ MSAN between the MWNTs renders strong support to our finding 5 of an augmented parallel network formation of microcapacitors upon compatibilization. 6 In summary, the dielectric response of the biphasic system and its constituents in combina- 7 tion with knowledge of the interfacial dynamics is sufficient to predict the interfacial capac- 8 itance and the network formation of the microcapacitors. Interestingly, the phenomenon is 9 a signature of the connectivity of the biphasic morphology, even though it is fundamentally 10 dissimilar to increasing conductivity. In summary, the results presented in Figure 9 establish 11 a novel methodology for predicting the interfacial capacitance and probing the connectivity 12 of microcapacitors in complex systems. 


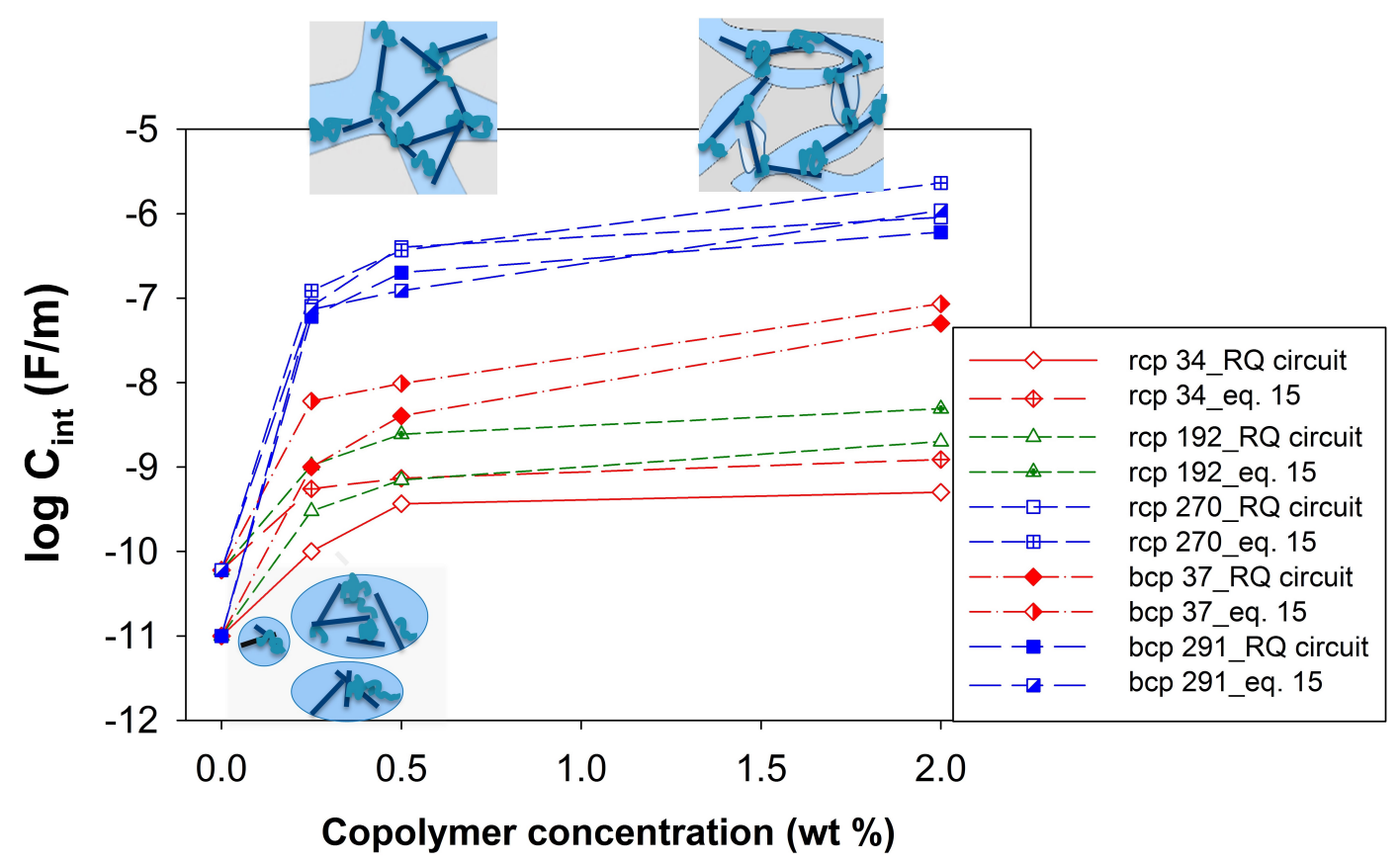

Figure 9. Interfacial capacitance of the MWNTs-entrapped P $\alpha$ MSAN network obtained from Figure 5 (designated as RQ circuit), and from eq 16 versus rcp/bcp concentration for bi-phasic 40/60 P $\alpha$ MSAN/PMMA blends with $0.5 \mathrm{wt} \%$ MWNTs at $220^{\circ} \mathrm{C}$. The interlayer model was used to determine $\phi_{f}$ and the fluctuation induced tunneling model was used to determine $\delta$ in eq 16. Inset: Schematic of the biphasic morphology with increasing MWNT interfacial capacitance.

\section{Conclusions}

We report for the first time an effect of copolymers architecture, molecular weight and concentration on the dielectric MWNT-P $\alpha$ MSAN interfacial capacitance in phase separated 40/60 (wt\%/ wt\%) P $\alpha$ MSAN/PMMA blends with 0.5 wt\% MWNTs. Compatibilization was achieved upon interfacial segregation of a block (bcp) or random copolymer (rcp) 5 of PS-PMMA, while the MWNTs selectively localized in the P $\alpha$ MSAN phase. The AC 6 conductivity and dielectric properties reflect local motions of charge carriers governed by 7 the mesoscopic length scales of the materials. At high frequencies (above $1 \mathrm{MHz}$ ) the 8 electrons hop between the adjacent MWNTs, whereas at intermediate frequencies, the 9 electrons of the MWNTs tunnel through the barriers imposed by the entrapped P $\alpha$ MSAN. $\quad 10$ 
The latter phenomenon results in an interfacial dielectric relaxation peak, similar to that 1 in MWNTs-P $\alpha$ MSAN composites. To further quantify the MWNT-P $\alpha$ MSAN interfacial 2 dielectric properties in the blends, an equivalent electrical circuit was developed and its 3 parameters were determined from the Nyquist plots. Adjacent MWNTs entrapping a thin 4 dielectric layer of $\mathrm{P} \alpha \mathrm{MSAN}$ form a microcapacitor, represented by an RC element. The total 5 interfacial capacitance increases with addition of copolymer, whereas the total interfacial 6 resistance decreases. The effect of various copolymers on the physical characteristics 7 of the microcapacitor network, i.e. the gap spacing of the capacitors and the volume 8 fraction of the entrapped P $\alpha$ MSAN chains between the MWNTs was elucidated. Using 9 the fluctuation induced tunneling (FIT) model, the gap spacing was calculated from the 10 interfacial relaxation time, obtained from either the product of the interfacial resistance 11 and capacitance or from HN fitting of the "conduction free" dielectric spectra, giving 12 similar results. The volume fraction of entrapped P $\alpha \mathrm{MSAN}$ chains was estimated using the 13 dielectric interlayer model. The effect of the copolymer on the gap spacing was negligible, 14 whereas the volume fraction of entrapped P $\alpha$ MSAN chains increased by 4 decades upon 15 effective compatibilization. This was attributed to an increase in the connectivity of the 16 dispersed microcapacitors of entrapped P $\alpha$ MSAN between MWNTs without changing the 17 characteristics of each capacitor. Combining the thickness of each microcapacitor and 18 the volume fraction of entrapped P $\alpha \mathrm{MSAN}$ contributing to the microcapacitor network 19 allowed to estimate the total interfacial capacitance. A remarkable agreement between 20 the total interfacial capacitance obtained using aforementioned method and the interfacial 21 capacitance measured as a fitting parameter in the Nyquist fitting of the impedance 22 response was obtained. In conclusion, effective compatibilization led to an increase in ${ }_{23}$ the interfacial capacitance originating from the microcapacitor network of MWNTs in ${ }_{24}$ the P $\alpha$ MSAN phase. It is known that the state of percolation and the local organization 25 of the MWNTs governs the performance of a material, like its resistance against crack 26 initiation and crack propagation. ${ }^{53}$ Our findings can be used to independently tune the ${ }_{27}$ 
MWNT network percolation (via the copolymers) as well as the inter-MWNT distance 1 (via the MWNT concentration) allowing to tailor physical properties governed by various 2 length scales of the material. In addition, the developed characterization approach allows 3 to separately analyze the local microcapacitor properties as well as their connectivity. $\quad 4$

\section{Acknowledgement}

This research has been partially funded by GOA/09/002. Ruth Cardinaels is indebted to 7 the Research Foundation Flanders (FWO) for a Post-doctoral Fellowship at KU Leuven. 8 


\section{References}

(1) Van Puyvelde, P.; Velankar, S.; Moldenaers, P. Rheology and Morphology of Com- 2 patibilized Polymer Blends. Current Opinion in Colloid \& Interface Science 2001, 6, 3 457-463.

(2) Dai, C. A.; Dair, B. J.; Dai, K. H.; Ober, C. K.; Kramer, E. J.; Hui, C. Y.; Jelinski, L. W. $\quad 5$ Reinforcement of Polymer Interfaces with Random Copolymers. Physical Review Letters 6 1994, 73, 2472-2475.

(3) Eastwood, E. A.; Dadmun, M. D. Multiblock Copolymers in the Compatibilization 8 of Polystyrene and Poly(methyl methacrylate) Blends: Role of Polymer Architecture. 9 Macromolecules 2002, 35, 5069-5077.

(4) Van Hemelrijck, E.; Van Puyvelde, P.; Macosko, C. W.; Moldenaers, P. The Effect of ${ }_{11}$ Block Copolymer Architecture on the Coalescence and Interfacial Elasticity in Com- 12 patibilized Polymer Blends. Journal of Rheology 2005, 49, 783-798.

(5) Macosko, C.; Guegan, P.; Khandpur, A. K.; Nakayama, A.; Marechal, P.; Inoue, T. ${ }_{14}$ Compatibilizers for Melt Blending: Premade Block Copolymers. Macromolecules 1996, 15 29, 5590-5598.

(6) Barham, B.; Fosser, K.; Voge, G.; Waldow, D.; Halasa, A. Phase separation kinetics of ${ }_{17}$ a polymer blend modified by random and block copolymer additives. Macromolecules 18 2001, 34, 514-521.

(7) Bharati, A.; Cardinaels, R.; Van der Donck, T.; Seo, J. W.; Wübbenhorst, M.; Molde- ${ }_{20}$ naers, P. Tuning the Phase Separated Morphology and resulting Electrical Conductivity $\quad 21$ of Carbon Nanotube Filled Blends: Compatibilization by a Random or Block Copoly- 22 mer. Polymer 2017, 108, 483-492. 
(8) Barlow, J.; Paul, D. Mechanical compatibilization of immiscible blends. Polymer Engi- $\quad 1$ neering \&5 Science 1984, 24, 525-534.

(9) Ho, C.-H.; Wang, C.-H.; Lin, C.-I.; Lee, Y.-D. Synthesis and characterization of TPO- 3 PLA copolymer and its behavior as compatibilizer for PLA/TPO blends. Polymer 4 2008, 49, 3902-3910.

(10) Xu, Y.; Thurber, C. M.; Macosko, C. W.; Lodge, T. P.; Hillmyer, M. A. Poly (methyl 6 methacrylate)-block-polyethylene-block-poly (methyl methacrylate) Triblock Copoly- 7 mers as Compatibilizers for Polyethylene/Poly (methyl methacrylate) Blends. Indus- 8 trial \& Engineering Chemistry Research 2014, 53, 4718-4725.

(11) Kulasekere, R.; Kaiser, H.; Ankner, J.; Russell, T.; Brown, H.; Hawker, C.; Mayes, A. ${ }_{10}$ Homopolymer Interfaces Reinforced with Random Copolymers. Macromolecules 1996, 11 29, 5493-5496.

(12) Kremer, F.; Schönhals, A. Broadband Dielectric Spectroscopy; Springer, 2003.

(13) Bharati, A.; Wübbenhorst, M.; Moldenaers, P.; Cardinaels, R. Effect of Compatibiliza- ${ }_{14}$ tion on Interfacial Polarization and Intrinsic Length Scales in Biphasic Polymer Blends ${ }_{15}$ of P $\alpha$ MSAN and PMMA: A Combined Experimental and Modeling Dielectric Study. ${ }_{16}$ Macromolecules 2016, 49, 1464-1478.

(14) Al-Saleh, M. H.; Sundararaj, U. An innovative method to reduce percolation threshold 18 of carbon black filled immiscible polymer blends. Composites Part A: Applied Science 19 and Manufacturing 2008, 39, 284-293.

(15) Bharati, A.; Cardinaels, R.; Wübbenhorst, M.; Moldenaers, P. Enhancing the Conduc- 21 tivity of Carbon-Nanotube Filled Blends by Tuning their Phase Separated Morphology 22 with a Copolymer. Polymer 2015, 79, 271-282. 
(16) Dang, Z.-M.; Yuan, J.-K.; Zha, J.-W.; Zhou, T.; Li, S.-T.; Hu, G.-H. Fundamentals, 1 processes and applications of high-permittivity polymer-matrix composites. Progress 2 in Materials Science 2012, 57, 660-723.

(17) Yuan, J.-K.; Yao, S.-H.; Dang, Z.-M.; Sylvestre, A.; Genestoux, M.; Bai, J. Giant Dielectric Permittivity Nanocomposites: Realizing True Potential of Pristine Carbon 5 Nanotubes in Polyvinylidene Fluoride Matrix through an Enhanced Interfacial Inter- 6 action. The Journal of Physical Chemistry C 2011, 115, 5515-5521.

(18) Fritzsche, J.; Lorenz, H.; Klüppel, M. CNT Based Elastomer-Hybrid-Nanocomposites $\quad 8$ with Promising Mechanical and Electrical Properties. Macromolecular Materials and 9 Engineering 2009, 294, 551-560.

(19) Yuan, J.; Luna, A.; Neri, W.; Zakri, C.; Schilling, T.; Colin, A.; Poulin, P. Graphene ${ }_{11}$ liquid crystal retarded percolation for new high-k materials. Nature communications 12 $\mathbf{2 0 1 5}, 6,8700-8700$.

(20) Zhang, J.; Mine, M.; Zhu, D.; Matsuo, M. Electrical and dielectric behaviors and 14 their origins in the three-dimensional polyvinyl alcohol/MWCNT composites with low 15 percolation threshold. Carbon 2009, 47, 1311-1320.

(21) Laun, H. M. Rheological and Mechanical Properties of Poly(alpha-methylstyrene- ${ }_{17}$ co-acrylonitrile)/Poly(methyl methacrylate) Blends in Miscible and Phase Separated 18 Regimes of Various Morphologies - I. Characterization of Constituents, Blend Prepara- 19 tion, and Overview on Blend Morphology (Technical Report). Pure and Applied Chem- 20 istry 1998, 70, 1547-1566.

(22) Bose, S.; Cardinaels, R.; Özdilek, C.; Leys, J.; Seo, J. W.; Wübbenhorst, M.; Mold- ${ }^{22}$ enaers, P. Effect of Multiwall Carbon Nanotubes on the Phase Separation of Concen- ${ }_{23}$ trated Blends of Poly [( $\alpha$-methyl styrene)-co-acrylonitrile] and Poly (methyl methacry- 24 
late) as Studied by Melt Rheology and Conductivity Spectroscopy. European Polymer Journal 2014, 53, 253-269.

(23) Wübbenhorst, M.; van Turnhout, J. Analysis of Complex Dielectric Spectra. I. One- 3 Dimensional Derivative Techniques and Three-Dimensional Modelling. Journal of Non- 4 Crystalline Solids 2002, 305, 40-49.

(24) Steeman, P.; Maurer, F.; Van Turnhout, J. Dielectric Properties of Blends of Polycar- 6 bonate and Acrylonitrile-Butadiene-Styrene Copolymer. Polymer Engineering \& Sci- 7 ence 1994, 34, 697-706.

(25) Schmidt-Rohr, K.; Kulik, A.; Beckham, H.; Ohlemacher, A.; Pawelzik, U.; Boeffel, C.; 9 Spiess, H. W. Molecular Nature of the. beta. Relaxation in Poly (methyl methacrylate) 10 Investigated by Multidimensional NMR. Macromolecules 1994, 27, 4733-4745. 11

(26) Madbouly, S. A. Broadband Dielectric Spectroscopy for Poly (methyl methacry- 12 late)/Poly ( $\alpha$-methyl styrene-co-acrylonitrile) Blend. Polymer Journal 2002, 34, 515- 13 522.

(27) Bose, S.; Özdilek, C.; Leys, J.; Seo, J. W.; Wübbenhorst, M.; Vermant, J.; Molde- ${ }^{15}$ naers, P. Phase Separation as a Tool to Control Dispersion of Multiwall Carbon Nan- 16 otubes in Polymeric Blends. ACS Applied Materials \&J Interfaces 2010, 2, 800-807. 17

(28) Havriliak, S.; Havriliak, S. J. Dielectric and Mechanical Relaxation in Materials: Anal- ${ }_{18}$ ysis, Interpretation, and Application; Hanser New York, 1997.

(29) Vogel, H. Das temperaturabhängigkeitsgesetz der viskosität von flüssigkeiten. Phys. Z 20 1921, 22, 645-646.

(30) Fulcher, G. S. Analysis of Recent Measurements of the Viscosity of Glasses. Journal of ${ }_{22}$ the American Ceramic Society 1925, 8, 339-355. 
(31) Tammann, G.; Hesse, W. Die Abhängigkeit der Viscosität von der Temperatur bie 1 unterkühlten Flüssigkeiten. Zeitschrift für Anorganische und Allgemeine Chemie 1926, 2 $156,245-257$.

(32) Bello, A.; Laredo, E.; Marval, J. R.; Grimau, M.; Arnal, M. L.; Müller, A. J.; 4 Ruelle, B.; Dubois, P. Universality and percolation in biodegradable poly $\left(\varepsilon-{ }_{5}\right.$ caprolactone)/multiwalled carbon nanotube nanocomposites from broad band alter- 6 nating and direct current conductivity at various temperatures. Macromolecules 2011, 7 44, 2819-2828.

(33) Meier, J. G.; Mani, J. W.; Klüppel, M. Analysis of Carbon Black Networking in Elas- 9 tomers by Dielectric Spectroscopy. Physical Review B 2007, 75, 054202.

(34) Dyre, J. C. The Random Free-Energy Barrier Model for Ac Conduction in Disordered ${ }_{11}$ Solids. Journal of Applied Physics 1988, 64, 2456-2468.

(35) Lvovich, V. F. Impedance Spectroscopy: Applications to Electrochemical and Dielectric ${ }_{13}$ Phenomena; John Wiley \& Sons, 2012.

(36) Hsu, C.; Mansfeld, F. Technical note: concerning the conversion of the constant phase ${ }_{15}$ element parameter Y0 into a capacitance. Corrosion 2001, 57, 747-748.

(37) Jiang, M.-J.; Dang, Z.-M.; Bozlar, M.; Miomandre, F.; Bai, J. Broad-frequency Dielec- ${ }_{17}$ tric behaviors in Multiwalled Carbon Nanotube/Rubber Nanocomposites. Journal of 18 Applied Physics 2009, 106, 084902.

(38) Buldum, A.; Lu, J. P. Contact Resistance between Carbon Nanotubes. Physical Review 20 B 2001, 63, 161403.

(39) Tersoff, J. Contact Resistance of Carbon Nanotubes. Applied Physics Letters 1999, 74, 22 $2122-2124$. 
(40) Starr, F. W.; Schrøder, T. B.; Glotzer, S. C. Molecular Dynamics Simulation of a 1 Polymer Melt with a Nanoscopic Particle. Macromolecules 2002, 35, 4481-4492. 2

(41) Nan, C.-W.; Shen, Y.; Ma, J. Physical Properties of Composites near Percolation. 3 Annual Review of Materials Research 2010, 40, 131-151.

(42) Pötschke, P.; Dudkin, S. M.; Alig, I. Dielectric Spectroscopy on Melt Processed 5 Polycarbonate-Multiwalled Carbon Nanotube Composites. Polymer 2003, 44, 5023- 6 5030.

(43) Fritzsche, J.; Klüppel, M. Structural Dynamics and Interfacial Properties of Filler- 8 reinforced Elastomers. Journal of Physics: Condensed Matter 2011, 23, 035104.

(44) Kilbride, B.; Coleman, J.; Fraysse, J.; Fournet, P.; Cadek, M.; Drury, A.; Hutzler, S.; 10 Roth, S.; Blau, W. Experimental Observation of Scaling Laws for Alternating Current ${ }_{11}$ and Direct Current Conductivity in Polymer-Carbon Nanotube Composite Thin Films. $\quad 12$ Journal of Applied Physics 2002, 92, 4024-4030. 13

(45) Kymakis, E.; Amaratunga, G. A. Electrical Properties of Single-Wall Carbon Nanotube- ${ }_{14}$ Polymer Composite Films. Journal of Applied Physics 2006, 99, 084302.

(46) Sheng, P.; Sichel, E.; Gittleman, J. Fluctuation-induced tunneling conduction in 16 carbon-polyvinylchloride composites. Physical Review Letters 1978, 40, 1197.

(47) Ahmad, K.; Pan, W. Dramatic effect of multiwalled carbon nanotubes on the elec- ${ }_{18}$ trical properties of alumina based ceramic nanocomposites. Composites Science and 19 Technology 2009, 69, 1016-1021.

(48) Simmons, J. G. Generalized Formula for the Electric Tunnel Effect between Similar 21 Electrodes Separated by a Thin Insulating Film. Journal of Applied Physics 1963, 34, 22 1793-1803. 
(49) Steeman, P.; Maurer, F. An Interlayer Model for the Complex Dielectric Constant 1 of Composites: An Extension to Ellipsoidally Shaped Particles. Colloid and Polymer 2 Science 1992, 270, 1069-1079.

(50) Steeman, P.; Maurer, F.; Van Es, M. Dielectric Monitoring of Water Absorption in Glass-bead-filled High-density Polyethylene. Polymer 1991, 32, 523-530.

(51) Steeman, P.; Baetsen, J.; Maurer, F. Temperature Dependence of the Interfacial Di- 6 electric Loss process in Glass Bead-filled Polyethylene. Polymer Engineering 85 Science 7 1992, 32, 351-356.

(52) Sichel, E. K. Carbon Black-Polymer Composites: The Physics of Electrically Conducting 9 Composites; Marcel Dekker Inc, 1982; Vol. 3.

(53) Satapathy, B. K.; Weidisch, R.; Pötschke, P.; Janke, A. Tough-to-brittle Transition in 11 Multiwalled Carbon Nanotube (MWNT)/Polycarbonate Nanocomposites. Composites 12 Science and Technology 2007, 67, 867-879. 


\section{Table of Contents}

Dielectric Properties of Phase Separated Blends containing a 2 Microcapacitor Network of Carbon Nanotubes: Compatibilization 3 by a Random or Block Copolymer

Avanish Bharati, Michael Wübbenhorst, Paula Moldenaers*, Ruth Cardinaels

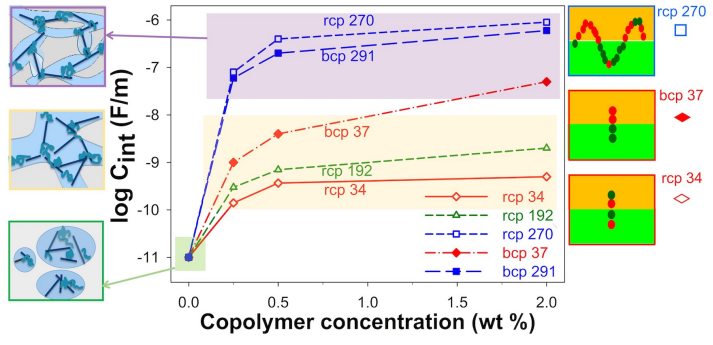

